Nuclear Physics B205[FS5] (1982) 545-577

(c) North-Holland Publishıng Company

\title{
GAUGE FIELD THERMODYNAMICS FOR THE SU(2) YANG-MILLS SYSTEM
}

\author{
J ENGELS, F KARSCH and H SATZ \\ Fakultat fur Physı, Universitat Bielefeld, Germanv \\ I MONTVAY \\ II Institut fur Theoretische Physik ${ }^{\star}$, Universitat Hamburg, Germany
}

Received 11 February 1982

\begin{abstract}
After reviewing the euclidean formulation of the thermodynamics for quantum spin systems, we develop the corresponding formalısm for $\mathrm{SU}(N)$ gauge fields on the lattice The results are then evaluated for the SU(2) system, using Monte Carlo simulation on lattices of (space $\times$ temperature) size $10^{3} \times 2,3,4,5$ At high temperature, the system exhibits Stefan-Boltzmann behaviour, with three gluonic colour degrees of freedom At $T_{\mathrm{c}} \approx 43 \Lambda_{\mathrm{L}}(215 \mathrm{MeV})$, the transition to "hadronc" behaviour occurs, signalled by a sharp peak in the specific heat From the behaviour below the deconfinement transition $\left(T<T_{\mathrm{c}}\right)$, we obtain $m_{\mathrm{G}} \approx 200 \Lambda_{\mathrm{L}}(1000 \mathrm{MeV})$ for the mass of the lowest gluonium state (glueball)
\end{abstract}

\section{Introduction}

The thermodynamics of strongly interacting matter has been a subject of much interest for a long time. Even before the introduction of the quark structure of hadrons, it had provided indications for some type of critical behaviour [1]; once hadrons were considered as composite objects, it was conjectured almost immediately that at sufficiently high densities or temperatures, strongly interacting matter should undergo a phase transition from a state of interacting hadrons to a quark-gluon plasma [2]. The advent of quantum chromodynamics led to the hope that both the two-phase nature and the transition could be derived from one basic theory, particularly the strong coupling results in the lattice formulation of QCD provided further support for this hope [3]. In the past two years, the Monte Carlo simulation of lattice QCD [4] has turned out to be a very effective way to study this question, at least for the case of pure Yang-Mills systems: interacting gluon matter does exhibit a deconfining transition [5-10], which separates a colour-screened gluon gas at high temperatures [7-9] from a gluonium gas at low temperatures [10].

\footnotetext{
* Supported by the Bundesministerium fur Forschung and Technologie, Bonn
} 
The alm of this paper is to present a comprehensive and systematic development of the thermodynamics of matter described by SU(2) Yang-Mills theory. Our startıng point will be the euclidean lattice formulation of QCD [11], and we shall use the Monte Carlo simulation of the lattice problem to perform the evaluation [4]. What we obtain in this way is on one hand a generalization of black-body thermodynamics to the case of interacting fields, on the other hand a model for the thermodynamics of QCD systems. For this it is a model only because of the restriction to a pure Yang-Mills system; the extension to $\mathrm{SU}(3)$ is straightforward and does not seem to induce significant changes in behaviour $[8,9]$. Work on the inclusion of Fermi fields in lattice studies is presently being pursued vigorously by several groups [12], and in the foreseeable future finite temperature thermodynamics may well be extended in this direction.

The paper is organized in the following way. In sect. 2, we study simple quantum spin systems, both in conventional and in gauge-invariant form, introducing here the euclidean lattice form of thermodynamics to be employed later for the Yang-Mills system. This allows us to illustrate in the case of physically quite transparent instances the main features of the transition from hamiltonian to euclidean thermodynamics; moreover, the gauge-invariant quantum Ising model already exhıbıts a behaviour very similar to that of the Yang-Mills field. In sect. 3, we then consider the free Bose field in euclidean lattice formulation. Since for this case the continuum limit is well known, we can use it to estımate the effect of finite lattice size in our calculations, and to determine lattice parameters optimizing the approxımation. In sect. 4 we formulate euclidean lattice thermodynamics for Yang-Mills fields, and in sect. 5 we present the basic thermodynamic quantities for the SU(2) case, at high temperature, in the deconfinement region and in the gluonium regime.

\section{Euclidean thermodynamics for quantum spin systems}

In the conventional formulation of statistical mechanics, the starting point is the partition function

$$
Z(\beta)=\operatorname{Tr} \mathrm{e}^{-\beta H},
$$

defined in terms of the hamiltonian $H$ of the system; $\ln Z(\beta)$ counts the number of possible states at a given physical temperature $T=\beta^{-1}$. For field-theoretic systems, $H$ becomes the three-dimensional integral over the hamiltonian density $\mathcal{H}(x)$,

$$
H=\int \mathrm{d}^{3} x \mathcal{H}(x)
$$

with $\mathcal{H}(x)$ given in terms of fields $A(x)$. It was shown [13] that the partition function (2.1), (2.2) can be reformulated as a Feynman functional integral involving 
the euclidean action:

$$
\begin{aligned}
& Z(\beta)=N(\beta) \int[\mathrm{d} A] \mathrm{e}^{-S(\beta)}, \\
& S(\beta)=-\int_{0}^{\beta} \mathrm{d} \tau \int \mathrm{d}^{3} x \mathcal{L}(\boldsymbol{x}, \tau) .
\end{aligned}
$$

Here the lagrangian density $\mathcal{E}\left(\boldsymbol{x}, x_{0}\right)$ is taken at imaginary tıme $x_{0}=i \tau$, with periodic boundary conditions $A(\boldsymbol{x}, 0)=A(\boldsymbol{x}, \beta)$ for bosonic fields; $N(\beta)$ is a normalizıng factor.

Since the form (2.3), (2.4) will provide the startıng point for lattice thermodynamics, we shall begin by considering simple quantum spin systems with a given hamıltonian formulation. By treating these in the euclidean approach, we can familiarize ourselves with the new features this implies.

\section{THE ISING MODEL IN A TRANSVERSE FIELD}

The hamiltonian of the one-dimensıonal Ising model with a transverse field $\gamma$ ("quantum Ising model") is given by [14]

$$
H=-\sum_{k=1}^{N} \sigma_{3}(k) \sigma_{3}(k+1)-\gamma \sum_{k=1}^{N} \sigma_{1}(k),
$$

with periodic boundary conditions, $\sigma_{3}(N+1)=\sigma_{3}(1)$, and with the spin-spin interaction energy taken as unity. The corresponding partition function,

$$
Z_{N}(\beta, \gamma)=\operatorname{Tr}_{N} \mathrm{e}^{-\beta H}
$$

at temperature $T=\beta^{-1}$ can be evaluated analytically for $N \rightarrow \infty$, giving

$$
\begin{aligned}
\lim _{N \rightarrow \infty} \frac{1}{N} \ln Z_{N}(\beta, \gamma) & =\frac{1}{2 \pi} \int_{0}^{2 \pi} \mathrm{d} \phi \ln (2 \cosh [\beta \Lambda(\phi, \gamma)]) \\
\Lambda(\phi, \gamma) & \equiv\left(1+\gamma^{2}-2 \gamma \cos \phi\right)^{1 / 2}
\end{aligned}
$$

It is analytic for all $T>0$; at $T=0$, we obtain

$$
\varepsilon_{0}(\gamma)=\frac{(1+\gamma)}{\pi} E\left(\frac{4 \gamma}{(1+\gamma)^{2}}\right)
$$

for the energy per spin; here $E(x)$ is a complete elliptic integral of the second kind, which is non-analytic at $x=1$, with $\mathrm{d} E / \mathrm{d} x$ diverging there. This results in a critical 

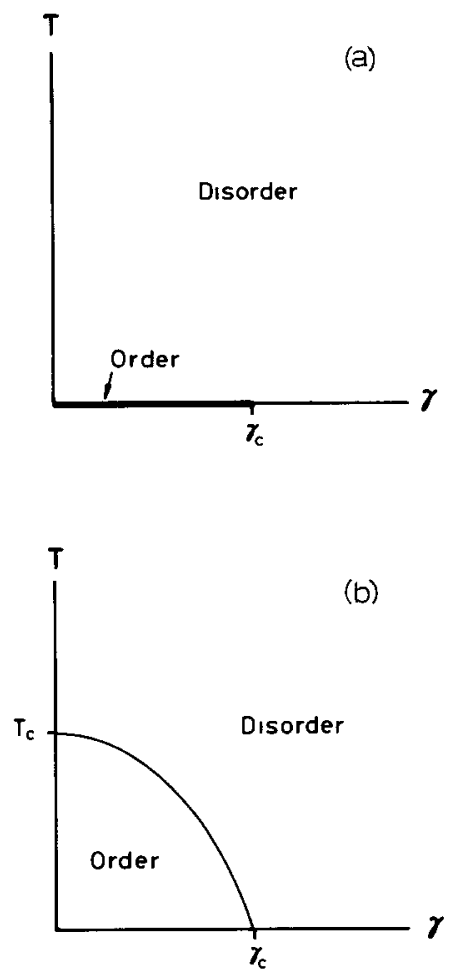

Fig 1 Phase diagrams for (a) one-dimensional and (b) higher-dimensional Isıng models with a transverse field of coupling strength $\gamma$

point at $\gamma_{\mathrm{c}}=1$; the phase diagram for the system is shown in fig. 1a. The nature of the two phases is readily seen: at $\gamma=0$, we recover the usual one-dimensional Ising model, which is disordered everywhere except at $T=0$, where the system is in one of the two degenerate ground states of completely aligned spins ("up" or "down"). Turning on the transverse field tends to disturb the alignment, and for $\gamma \geqslant \gamma_{c}$, the degeneracy is removed and the order disappears. In higher dimensions, the corresponding problem has not been solved in closed form [15]. The phase structure is nevertheless apparent; it is shown in fig. $1 \mathrm{~b}$.

We now want to express the one-dimensional case in euclidean formulation Using the generalized Trotter formula [16], the trace (2.6) can be written in the form

$$
Z_{N}(\beta, \gamma)=\lim _{M \rightarrow \infty} Z_{N, M}\left(a_{\beta}, \gamma\right), \quad a_{\beta} M=\beta \text { fixed }
$$

where

$$
Z_{N, M}\left(a_{\beta}, \gamma\right)=\left[\frac{1}{2} \sinh \left(2 a_{\beta} \gamma\right)\right]^{N M / 2} Z_{N, M}^{\mathrm{E}}\left(a_{\beta}, \gamma\right)
$$


and where

$$
\begin{aligned}
Z_{N, M}^{\mathrm{E}}\left(a_{\beta}, \gamma\right)=\operatorname{Tr}_{N, M} \exp \left\{\sum _ { j = 1 } ^ { N } \sum _ { k = 1 } ^ { M } \left[a_{\beta} \sigma_{3}(j, k) \sigma_{3}(j+1, k)\right.\right. \\
\left.\left.+g\left(a_{\beta}, \gamma\right) \sigma_{3}(j, k) \sigma_{3}(j, k+1)\right]\right\}
\end{aligned}
$$

is the partition function of the anisotropic, two-dimensional classical Ising model. The quantum problem (2.5), (2.6), where $H$ contains non-commuting terms, has thereby been reformulated as a classical problem in one more dimension corresponding to the temperature $\beta=M a_{\beta}$. The hamiltonian form $(2.5),(2.6)$ and the euclidean form (2.11), (2.12) become identical in the temperature continuum limit $M \rightarrow \infty$ at fixed $\beta=M a_{\beta}$. The vanishing of $a_{\beta}$ in this limit must be compensated by a suitable change in the temperature coupling $g\left(a_{\beta}, \gamma\right)$, such as to leave physics invariant. With $H$ explicitly given, one finds

$$
g\left(a_{\beta}, \gamma\right)=\frac{1}{2} \ln \operatorname{coth}\left(a_{\beta} \gamma\right)
$$

from the Trotter formula [16].

The two-dimensional classical Isıng model (2.12) has the well-known Onsager phase transition at

$$
\sinh 2 a_{\beta} \sinh 2 g\left(a_{\beta}, \gamma\right)=1
$$

in the limit $a_{\beta} \rightarrow 0$ this implies $\gamma_{\mathrm{c}}=1$, in accord with the result from eq. (2.9).

The free energy per spin,

$$
f(\beta, \gamma)=\frac{-1}{N \beta} \ln Z_{N}(\beta, \gamma)
$$

can with eq. (2.11) be written

$$
\begin{aligned}
f(\beta, \gamma) & =\lim _{M \rightarrow \infty}\left\{f_{M}^{\mathrm{E}}\left(a_{\beta}, \gamma\right)-\frac{M}{2 \beta} \ln \left[\frac{1}{2} \sinh \left(2 a_{\beta} \gamma\right)\right]\right\}, \\
f_{M}^{\mathrm{E}}\left(a_{\beta}, \gamma\right) & \equiv \frac{-1}{N \beta} \ln Z_{N, M}^{\mathrm{E}}\left(a_{\beta}, \gamma\right) .
\end{aligned}
$$

To understand this shift between hamıltonian and euclidean free energy, we note 
that from eqs. (2.11), (2.12)

$$
\begin{aligned}
& \lim _{M \rightarrow \infty} \frac{1}{N} \ln Z_{N, M}\left(a_{\beta}, \gamma\right)= \lim _{M \rightarrow \infty} \frac{1}{N} \ln \operatorname{Tr}_{N, M} \\
& \times \exp \left\{\sum _ { J = 1 } ^ { N } \sum _ { k = 1 } ^ { M } \left[a_{\beta} \sigma_{3}(j, k) \sigma_{3}(j+1, k)\right.\right. \\
&\left.\left.\quad-g\left(a_{\beta}, \gamma\right)\left(1-\sigma_{3}(j, k) \sigma_{3}(j, k+1)\right)\right]\right\} ;
\end{aligned}
$$

the additional term in eq. (2.16) shifts $\sigma_{3} \sigma_{3} \rightarrow-1+\sigma_{3} \sigma_{3}$ in the temperature direction, thus normalizing the ground-state contribution of that part to zero.

We summarize. the quantum problem of a one-dimensional Ising model in a transverse field can be restated as a classical problem with an additional temperature dimension. The two forms become equivalent in the temperature continuum limit, provided the coupling $g\left(a_{\beta}, \gamma\right)$ in the temperature direction is suitably changed when the associated lattice spacing $a_{\beta}$ is decreased, and provided the free energy is shifted to assure the correct vacuum structure.

Before going on to the gauge-ınvariant case, let us briefly note the connection between phase structure and duality for the model just studied [17].

The hamiltonian (2.5) is invariant under simultaneous inversion of all spins (global symmetry); as a consequence, one can define a local order parameter, the magnetization

$$
m \equiv\left\langle\sigma_{3}\right\rangle
$$

measurnng the average spin value per site. It is finite for $\gamma<1$ and vanishes for $\gamma>1$, thus specifying the phase of the system.

Consider now the same model on the dual lattice, whose sites correspond to the links of the original lattice. The spin operators $\sigma_{1}$ and $\sigma_{3}$ are replaced by the operators

$$
\begin{aligned}
& \mu_{1}(k)=\sigma_{3}(k) \sigma_{3}(k+1), \\
& \mu_{3}(k)=\prod_{n<k} \sigma_{1}(n),
\end{aligned}
$$

which satisfy the same algebra. The hamiltonian (2.5) retains its form under the duality transformation $\sigma \rightarrow \mu$ :

$$
\gamma H\left(\mu, \gamma^{-1}\right)=H(\sigma, \gamma)
$$


it is self-dual. Since $\mu_{3}(k)$ flips all spins to the left of $k$ on the original lattice,

$$
\tilde{m} \equiv\left\langle\mu_{3}\right\rangle
$$

vanıshes in the ordered phase and is finite in the disordered. Thus $\gamma=1$ is the critical point, since there order and disorder become indistınguishable.

For a self-dual, globally symmetric model such as (2.5), we can thus determine the phase structure either by order parameter or by duality relatıon.

\section{THE GAUGE-INVARIANT ISING MODEL IN A TRANSVERSE FIELD}

The hamiltonian of the gauge-invariant Isıng model in three space dimensions and with a transverse field $\gamma$ is given by [17-19]

$$
H=-\sum_{\{\mathbf{P}\}} \sigma_{3}(l) \sigma_{3}(J) \sigma_{3}(k) \sigma_{3}(l)-\gamma \sum_{\{\mathbf{L}\}} \sigma_{1}(l) .
$$

The spins are now associated to the links between any two adjacent sites. The first sum runs over all primitive squares (plaquettes) of the lattice, the second over all links. We note that in contrast to the global symmetry of the normal Ising model, the hamiltonian (2.24) remains invariant even if only the spins on all lınks connected to any one given site are flipped (local symmetry). As a consequence, one cannot define local order parameters such as the magnetization (2.19). from a state of completely aligned spins we can by gauge transformation go to one with flipped spins around one or more sites. For physical states, $\left\langle\sigma_{3}\right\rangle$ must therefore vanısh everywhere [20]. The phase structure of systems such as (2.24) thus has to be specified differently.

In the normal Ising model, correlations between any two spins vanish exponentrally with the distance of separation, if the system is in the disordered phase. $\left\langle\sigma_{3}(0) \sigma_{3}(k)\right\rangle \sim \exp (-k / \xi(T))$, where $\xi(T)$ is the correlation length. In the ordered phase, the system is magnetized, so that $\left\langle\sigma_{3}(0) \sigma_{3}(k)\right\rangle \sim\left\langle\sigma_{3}(0)\right\rangle^{2}$. Differences in the behaviour of correlations as a criterion for phase structure can be generalized to gauge-invariant models. The function $\left\langle\sigma_{3}(0) \sigma_{3}(k)\right\rangle$, just as $\left\langle\sigma_{3}\right\rangle$, is not gauge invariant and must therefore vanish identically. However, the plaquette average $\left\langle\sigma_{3}(l) \sigma_{3}(J) \sigma_{3}(k) \sigma_{3}(l)\right\rangle$, obtained by averaging over all primitive lattice squares, is gauge invariant, and so is the average over a product of $\sigma_{3}$ 's along a closed path $C$, of length $P$ and enclosing an area $A$. The behaviour of $C(A)=\prod_{i \in \mathrm{P}} \sigma_{3}(l)$ for $A \rightarrow \infty$ is found to differ for large and small $\gamma$ :

$$
\ln C(A) \sim \begin{cases}-A, & \gamma>1 \\ -P, & \gamma<1,\end{cases}
$$

for $A$ sufficiently large. Moreover, the hamiltonian (2.24) can be shown to be self-dual, with $\gamma=1$ as critical point for the ground state. Functional differences in 


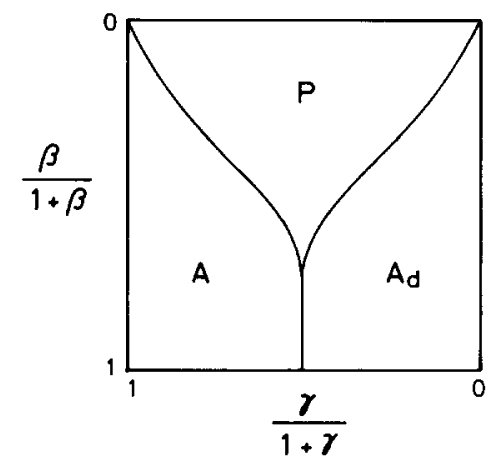

Fig 2 Phase diagram of the three-dimensional gauge-invariant Isıng model with a transverse field of coupling strength $\gamma$ [19] The notation is explained in the text

correlation measures such as $C(A)$ can thus be used to map out the phase structure of gauge-invariant systems.

To obtain the full phase diagram [19], it is first noted that the area law region of eq. (2.25) decreases with increasing temperature. Further, on the dual lattice (constructed in a similar way as in subsect. 2.1 [17]), one fınds an analogous behaviour in $\gamma^{-1}$. We are thus led to three phases, as shown in fig. 2. In the region marked A, we have area-law behaviour on the lattice, perımeter-law behaviour on the dual lattice; in $\mathrm{A}_{\mathrm{d}}$, the situation is just the opposite. In the region $P$ finally, both lattice and dual lattice show a perimeter law decrease.

To understand the physical nature of these phases, we recall that an area-law behaviour implies for a pair of external sources immersed in the system a binding potential which increases linearly with the distance of separation (confinement). The perımeter behaviour, on the other hand, is connected to a Coulomb-type potential (non-confinement). Associating the link term of eq. (2.24) with an electric, the plaquette term with a magnetıc force field, we have in phase $A$ confinement of electric charges, in phase $A_{d}$ confinement of magnetic monopoles, and in phase $P$ complete deconfinement.

Let us now study this system in the euclidean formulation; we shall here encounter already many of the main features of the SU(2) Yang-Mills system.

As in subsect. 2.1, we use Trotter's formula to rewrite the partition function

$$
Z_{N}(\beta, \gamma)=\operatorname{Tr}_{N} \mathrm{e}^{-\beta H},
$$

where $H$ is given by eq. (2.24) and the trace 1 s over all physical states, 1.e., gauge-invariant states obeying Gauss' law. Analogous to eq. (2.11), (2.12) we obtain

$$
Z_{N, M}\left(a_{\beta}, \gamma\right)=\left[2^{-5 / 3} \sinh \left(2 a_{\beta} \gamma\right)\right]^{3 N^{3} M / 2} Z_{N, M}^{\mathrm{E}}\left(a_{\beta}, \gamma\right)
$$


with

$$
Z_{N, M}^{\mathrm{E}}\left(a_{\beta}, \gamma\right)=\sum_{\left\{\sigma_{3}= \pm 1\right\}} \exp \left\{a_{\beta} \sum_{\left\{\mathrm{P}_{\alpha}\right\}} \sigma_{3} \sigma_{3} \sigma_{3} \sigma_{3}+g\left(a_{\beta}, \gamma\right) \sum_{\left\{\mathrm{P}_{\beta}\right\}} \sigma_{3} \sigma_{3} \sigma_{3} \sigma_{3}\right\}
$$

as partition function of the four-dimensional anısotropic gauge-invariant Isıng model, with $N$ sites in each space direction and $M$ sites on the "temperature" axis, $\beta=M a_{\beta}$. The basic summation is over all links of the lattice; the sum $\left\{\mathrm{P}_{\sigma}\right\}$ runs over all plaquettes with only spacelike links, the $\operatorname{sum}\left\{\mathrm{P}_{\beta}\right\}$ over those with two space-like and two temperature-like links. The coupling constant is as in subsect. 2.1 given by

$$
g\left(a_{\beta}, \gamma\right)=\frac{1}{2} \ln \operatorname{coth}\left(a_{\beta} \gamma\right)
$$

and as before the connection between euclidean and hamiltonian forms becomes exact for $M \rightarrow \infty$,

$$
Z_{N}(\beta, \gamma)=\lim _{M \rightarrow \infty} Z_{N, M}\left(a_{\beta}, \gamma\right)
$$

at fixed $\beta=M a_{\beta}$, 1.e., in the temperature contınuum limit.

The energy density,

$$
\varepsilon(\beta, \gamma) \equiv-\frac{1}{N^{3}}\left(\frac{\partial \ln Z_{N}(\beta, \gamma)}{\partial \beta}\right)
$$

1s in the euclidean formulation on a finite lattice approximated by

$$
\varepsilon_{E}(\beta, \gamma)=-\frac{1}{N^{3} M}\left(\frac{\partial \ln Z_{N, M}^{\mathrm{E}}\left(a_{\beta}, \gamma\right)}{\partial a_{\beta}}\right)_{M}
$$

this also becomes exact for $M \rightarrow \infty$ at fixed $\beta$. Using eq. (2.27), (2.28), we obtain

$$
\varepsilon_{\mathrm{E}}(\beta, \gamma)=3\left[\left\langle P_{\mathrm{S}}\right\rangle+\frac{\partial g}{\partial a_{\beta}}\left\langle P_{\mathrm{T}}\right\rangle-1\right]
$$

with

$$
\begin{aligned}
\left\langle P_{\mathrm{S}}\right\rangle \equiv & \frac{1}{Z_{N, M}^{\mathrm{E}}\left(a_{\beta}, \gamma\right)} \sum_{\left\{\sigma_{3}= \pm 1\right\}}\left[\frac{\sum_{\left\{\mathrm{P}_{o}\right\}}\left(1-\sigma_{3} \sigma_{3} \sigma_{3} \sigma_{3}\right)}{3 N^{3} M}\right] \\
& \times \exp \left\{-a_{\beta} \sum_{\left\{\mathrm{P}_{\mathrm{o}}\right\}}\left(1-\sigma_{3} \sigma_{3} \sigma_{3} \sigma_{3}\right)-g\left(a_{\beta}, \gamma\right) \sum_{\left\{\mathrm{P}_{\beta}\right\}}\left(1-\sigma_{3} \sigma_{3} \sigma_{3} \sigma_{3}\right)\right\}
\end{aligned}
$$

as the average value of a space-like plaquette; similarly, $\left\langle P_{\mathrm{T}}\right\rangle$ is the average value of 
a space-temperature plaquette. From eq. (2.29), we have

$$
\frac{\partial g}{\partial a_{\beta}}=\frac{-\gamma}{\sinh 2 a_{\beta} \gamma}
$$

In eq. (2.33), (2.34), we have shifted from $\sigma_{3} \sigma_{3} \sigma_{3} \sigma_{3}$ to the "normalized" form $\left(1-\sigma_{3} \sigma_{3} \sigma_{3} \sigma_{3}\right)$ for all plaquettes. In the temperature part, this shift is contained in the Trotter formula, as we saw in subsect. 2.1; for space-like plaquettes, it is achreved by separating out the energy of a completely ordered configuration. For the specific heat per unit volume

$$
c_{V}(\beta, \gamma)=-\beta^{2}\left(\frac{\partial \varepsilon}{\partial \beta}\right)
$$

we obtain similarly

$$
c_{\mathrm{V}, \mathrm{E}}(\beta, \gamma)=-\frac{3 \beta^{2}}{M}\left[\frac{\partial}{\partial a_{\beta}}\left(\left\langle\mathrm{P}_{\mathrm{S}}\right\rangle+\left\langle\mathrm{P}_{\mathrm{T}}\right\rangle \frac{\partial g}{\partial a_{\beta}}\right)\right],
$$

as the euclidean lattice approximation.

Evaluating eq. (2.33) by Monte Carlo simulation on a $4^{4}$ lattice, we obtain at $\gamma=1.5$ the behaviour shown in fig. 3. Because of the small lattice, these results will

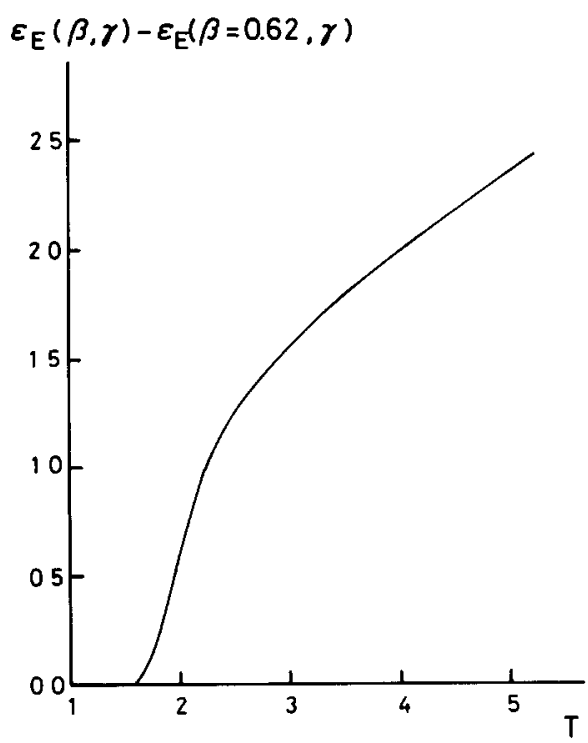

Fig 3 Energy density versus temperature for the gauge-invanant Ising model with a transverse field, calculated on a $4^{4}$ lattice at $\gamma=15$ 


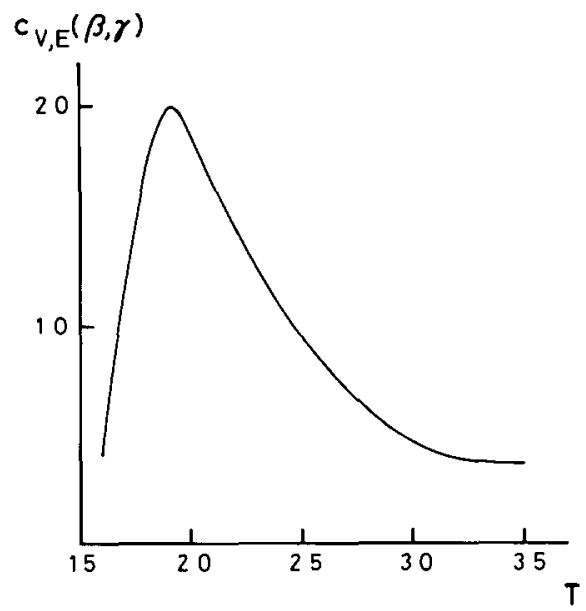

Fig 4 Specific heat per unit volume versus temperature obtained by differentiation from fig 3

presumably still contain finte size deviations; we have therefore in fig. 3 simply subtracted $\varepsilon_{\mathrm{E}}(\beta=0.62, \gamma=1.5)$ instead of the true vacuum contribution $\varepsilon_{\mathrm{E}}(T=0$, $\gamma=1.5)$. From fig. 2 we expect that increasing the temperature at this value of $\gamma$ will bring us from the (electric) confinement region through the deconfinement transition to a "free gas" phase. From the behaviour of the specific heat in fig. 4, obtained by differentiation from fig. 3 , this is indeed seen to be the case: the deconfinement transition is signalled, as in magnetic systems, by a singularity in the specific heat at temperature $T_{\mathrm{c}}$. Sufficiently far below $T_{\mathrm{c}}$, the energy density will approach the temperature-independent ground-state value $\varepsilon_{\mathrm{E}}(T=0, \gamma)$ with the spins frozen in a confined phase. At $T_{\mathrm{c}}, \varepsilon$ increases rather abruptly, and soon beyond $T_{\mathrm{c}}$ it behaves like a free spin system, $\varepsilon \sim T$.

To check our conclusion about deconfinement, we consider the expectation value of the thermal Wilson loop $L$, the product of $\sigma_{3}$ 's along the temperature direction at a fixed spatial link; because of the periodic boundary conditions imposed, this gives us a closed loop. Since $|\langle L\rangle|$ measures the free energy of an 1solated charge,

$$
|\langle L\rangle| \sim \mathrm{e}^{-\beta F},
$$

1t provides an order parameter for the system [5,6]: below $T_{\mathrm{c}}$ it should vanish, as a consequence of confinement, above $T_{\mathrm{c}}$ it should attain a finite value corresponding to the analogue of a Debye-screened charge. In fig. 5 we see this to be the case.

In summary we note that also the gauge-1nvariant quantum Ising model can be expressed as a classical problem in one more dimension, with exact equivalence in the temperature contınuum limit. The phase structure, because of gauge invariance, 




Fig 5 Thermal Wilson loop versus inverse temperature calculated on a $4^{4}$ (solid line) and a $6^{4}$ (dashed line) lattice

can no longer be specified by a local order parameter. Using instead a phase characterization by lattice loops, we have at low temperature a confined state, separated by a deconfinement transition, with singular specific heat, from a high temperature free gas phase. In sect. 5, we shall find a similar behaviour for the SU(2) Yang-Mills system; there the euclidean formulation of thermodynamics is the only general form we have. For a contınuum field theory we will, however, encounter questions of spatial in addition to temporal lattice structure.

\section{The free Bose field on a finite lattice}

In this section, we want to study the effect of the finite lattice size on the euchdean formulation of a system with a well-known contınuum limit: the free Bose field. As this problem, for both Bose and Fermı fields, is treated in detall elsewhere [21], we can be quite brief here.

The hamiltonıan of a non-interacting scalar Bose field $\varphi(x)$ for partıcles of mass $m$ is given by

$$
H=\frac{1}{2} \int \mathrm{d}^{3} x\left\{\pi^{2}(x)+(\nabla \varphi(x))^{2}+m^{2} \varphi^{2}(x)\right\} .
$$

The corresponding euclidean action is [13]

$$
S(\varphi)=\frac{1}{2} \int_{0}^{\beta} \mathrm{d} \tau \int \mathrm{d}^{3} x\left\{\left(\frac{\partial \varphi}{\partial \tau}\right)^{2}+(\nabla \varphi)^{2}+m^{2} \varphi^{2}\right\}
$$

where $\tau$ is the imaginary time. 
On a lattice with $N_{\sigma}^{3} \times N_{\beta}$ sites and lattice spacings $a_{\sigma}$ and $a_{\beta}$ in space and temperature directions, respectively, the action becomes

$$
\begin{aligned}
S(\varphi)=\frac{a_{\sigma}^{3} a_{\beta}}{2} \sum_{\alpha} & \left\{\sum_{\mu=1}^{3}\left(\frac{\varphi\left(x_{\alpha}+e_{\mu}\right)-\varphi\left(x_{\alpha}\right)}{a_{\sigma}}\right)^{2}\right. \\
& \left.+\left(\frac{\varphi\left(x_{\alpha}+e_{0}\right)-\varphi\left(x_{\alpha}\right)}{a_{\beta}}\right)^{2}+m^{2} \varphi^{2}\left(x_{\alpha}\right)\right\},
\end{aligned}
$$

where $x_{\alpha}=\left(\alpha_{0} a_{\beta}, \boldsymbol{\alpha} a_{\sigma}\right)$ denotes a lattice site and $e_{\mu}, \mu=0,1,2,3$, are the four lattice unit vectors. We impose periodic boundary conditions in all directions. The euclidean partition function $Z_{\mathrm{E}}$ is given in terms of the action (3.3)

$$
Z_{\mathrm{E}}\left(N_{\sigma}, N_{\beta}, a_{\sigma}, a_{\beta}\right)=N^{\prime} \int \prod_{\alpha} \mathrm{d} \varphi\left(x_{\alpha}\right) \exp [-S(\varphi)],
$$

with

$$
N^{\prime} \equiv\left[a_{\sigma}^{3} / 2 \pi a_{\beta}\right]^{N_{\sigma}^{3} N_{\beta} / 2}
$$

As in the previous section, it differs from the hamiltonian partition function $Z=\operatorname{Tr} \exp (-\beta H)$ by a ground-state correction factor.

The euclidean partition function can be evaluated to give [21]

$$
Z_{\mathrm{E}}\left(N_{\sigma}, N_{\beta}, a_{\sigma}, \xi\right)=\xi^{N_{\sigma}^{3} N_{\beta}} \prod_{q}\left\{\left(m a_{\sigma}\right)^{2}+4 \sum_{\mu=1}^{3} \sin ^{2}\left(\frac{1}{2} q_{\mu} a_{\sigma}\right)+4 \xi^{2} \sin ^{2}\left(\frac{1}{2} q_{0} a_{\beta}\right)\right\},
$$

with $\xi \equiv a_{\mathrm{o}} / a_{\beta}$, and with

$$
\left(N_{\beta} a_{\beta} / 2 \pi\right) q_{0}, \quad\left(N_{\sigma} a_{\sigma} / 2 \pi\right) q_{\mu}, \quad \mu=1,2,3
$$

running over positive and negative integer values, bounded by $\frac{1}{2} N_{\beta}$ and $\frac{1}{2} N_{\sigma}$, respectively. The free energy density $f$ is as in sect. 2 obtained by subtracting from

$$
f_{\mathrm{E}} \equiv-\ln Z_{\mathrm{E}} /\left(N_{\sigma}^{3} N_{\beta} a_{\sigma}^{3} a_{\beta}\right)
$$

the $T=0$ term,

$$
f_{0}=\lim _{N_{\beta} \rightarrow \infty} f_{\mathrm{E}}
$$

From eq. (3.6) we similarly obtain the energy density on the lattıce

$$
\varepsilon=\frac{-1}{V}\left(\frac{\partial \ln Z_{\mathrm{E}}}{\partial \beta}\right)_{V}-\varepsilon_{0}=\left(\xi^{2} / N_{\sigma}^{3} N_{\beta} a_{\sigma}^{4}\right)\left(\partial \ln Z_{\mathrm{E}} / \partial \xi\right)_{a_{\sigma}}-\varepsilon_{0} .
$$

Eq. (3.10) gives us $\varepsilon\left(N_{\sigma}, N_{\beta}, a_{\sigma}, \xi\right)$, normalized to zero for $N_{\beta} \rightarrow \infty$ with the other parameters fixed. 
In the continuum, the energy density of a massless ideal Bose gas is given by the well-known Stefan-Boltzmann form

$$
\varepsilon_{\mathrm{SB}}=\pi^{2} /\left(30 \beta^{4}\right) \text {. }
$$

Comparing this with eq. (3.10), we can study the effect of the finite lattice approximation. In fig. 6 , we show $\varepsilon / \varepsilon_{\mathrm{SB}}$ for different $N_{\sigma}, N_{\beta}$ and $\xi=1$. It is seen that the effect of finite lattice structure is by no means small. By letting $N_{\sigma} \rightarrow \infty$ at sufficiently large $N_{\beta}$, we can make $\varepsilon / \varepsilon_{\mathrm{SB}}$ as close to unity as desired. This is not true for $N_{\beta} \rightarrow \infty$ at fixed $N_{\sigma}$, however: increasing $N_{\beta}$ beyond a certain optimal value makes the lattice approximation at fixed $N_{\sigma}$ worse. The reason for this is that an increase of $N_{\beta}$ at fixed $a_{\sigma}, \xi$ and $N_{\sigma}$ is a decrease in temperature; but on a finite spatial lattice, we lose the low momentum modes which give the dominant contribution at low temperature. The best approxımation is thus always attained for $N_{\beta}$ as large as feasible with the condition $N_{\sigma} \gg N_{\beta}$.

In sect. 5 we shall see that the Monte Carlo evaluation of the SU(2) Yang-Mills system leads at very high temperatures to a behaviour very similar to what we have here, so that the values of $\varepsilon / \varepsilon_{\mathrm{SB}}$ displayed in fig. 6 seem to constitute a farly universal finite lattice size correction factor.

\section{Euclidean lattice thermodynamics for Yang-Mills fields}

In $\mathrm{SU}(N)$ Yang-Mills theory, the Lagrange density is given by

$$
\begin{aligned}
\varrho & =\frac{-1}{4} F_{\mu \nu}^{a} F_{a}^{\mu \nu}, \\
F_{\mu \nu}^{a} & =\partial_{\mu} A_{\nu}^{a}-\partial_{\nu} A_{\mu}^{a}-g f_{b c}^{a} A_{\mu}^{b} A_{\nu}^{c} .
\end{aligned}
$$

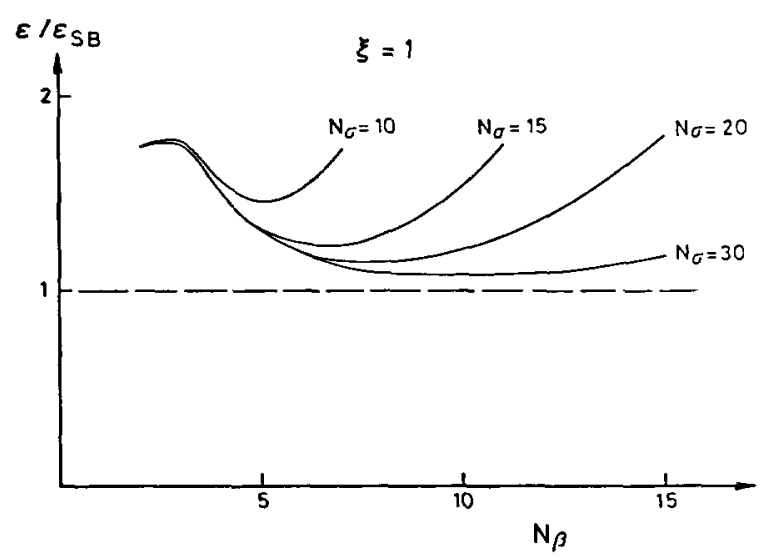

Fig 6 The rat1o $\varepsilon / \varepsilon_{\mathrm{SB}}$ for the 1 deal Bose gas versus $N_{\beta}$ for $\xi=1$ and various $N_{\mathrm{o}}$ 
The structure constants $f_{a b c}$ specify the commutation relations for the generators $\lambda_{a}$ of the $\mathrm{SU}(N)$ gauge group

$$
\left[\lambda_{a}, \lambda_{b}\right]=\imath f_{a b}^{c} \lambda_{c}
$$

the colour indices $(a, b, c)$ on the generators $\lambda_{a}$ and the gauge fields $A_{\mu}^{a}(x)$ run from 1 to $N^{2}-1$. The density (4.1), inserted in eq. (2.3), (2.4), gives us the partition function.

As in the previous section, the $x-\tau$ contınuum on which the fields $A_{\mu}^{a}(x)$ are defined, is replaced by a lattice with $N_{\sigma}\left(N_{\beta}\right)$ sites separated by spacings $a_{\sigma}\left(a_{\beta}\right)$ in the spatial (temperature) direction. The lattice is chosen to be closed on itself in all directions: in $\tau$ to assure the required periodicity, in $x$ to avold surface effects and for economy of calculation. To make certain that physical quantities in the contınuum limit will not depend on the lattice structure, we must introduce two coupling strengths, $g_{\sigma}$ and $g_{\beta}$, which replace the $g$ in eq (4.2). An independent variation in $a_{\sigma}$ and $a_{\beta}$ can then be compensated by suitably adjusting $g_{\sigma}$ and $g_{\beta}$ such as to keep physical quantities unchanged [22]. Finally we replace [11] the integration over the gauge fields in eq. (2.3) by one over the gauge group elements $U_{t j}$, associated to links between adjacent sites $\iota$ and $J$. With Wilson's form of the action, the partition function then becomes

$$
\begin{aligned}
Z_{\mathrm{E}}(\beta)= & \int \prod_{\text {links }} \mathrm{d} U_{\imath \jmath} \exp [-S(U)], \\
S(U)= & 2 N K_{\sigma} \sum_{\left\{\mathrm{P}_{\mathrm{a}}\right\}}\left(1-\frac{1}{N} \operatorname{Re} \operatorname{Tr} U_{l j} U_{j k} U_{k l} U_{l l}\right) \\
& +2 N K_{\beta} \sum_{\left\{\mathrm{P}_{\beta}\right\}}\left(1-\frac{1}{N} \operatorname{Re} \operatorname{Tr} U_{\imath} U_{j k} U_{k l} U_{l l}\right) .
\end{aligned}
$$

The sum $\left\{\mathrm{P}_{\sigma}\right\}$ runs over all purely space-lıke plaquettes $(l j k l),\left\{\mathrm{P}_{\beta}\right\}$ over those with two space-like and two temperature-like links. To recover the correct classical continuum action, we write

$$
U_{l j}=\exp \left\{-t\left(x_{i}-x_{j}\right)^{\mu} A_{\mu}\left(\frac{x_{i}+x_{j}}{2}\right)\right\}
$$

with $A_{\mu}(x)=\lambda_{a} A_{\mu}^{a}(x)$, and define the couplings

$$
K_{\sigma} \equiv \frac{1}{g_{\sigma}^{2}}\left(\frac{a_{\beta}}{a_{\sigma}}\right), \quad K_{\beta} \equiv \frac{1}{g_{\beta}^{2}}\left(\frac{a_{\sigma}}{a_{\beta}}\right)
$$


If in the limit of $\left(x_{t}-x_{f}\right) \rightarrow 0$

$$
g_{\sigma}^{2}=g_{\beta}^{2}=g^{2},
$$

then we can rescale $A_{\mu} \rightarrow g A_{\mu}$ and get back to eqs. (2.4) and (4.1), (4.2). Consider now the functions

$$
\begin{aligned}
& F_{\sigma}\left(a_{\sigma}, \xi\right)=g_{\sigma}^{-2}\left(a_{\sigma}, \xi\right) / g^{-2}\left(a_{\sigma}\right), \\
& F_{\beta}\left(a_{\sigma}, \xi\right)=g_{\beta}^{-2}\left(a_{\sigma}, \xi\right) / g^{-2}\left(a_{\sigma}\right),
\end{aligned}
$$

with $\xi=a_{\sigma} / a_{\beta}$. Since both are unity for $\xi=1$ as well as for $g^{2} \rightarrow 0$, we can expand about $g^{2}=0$ to get

$$
\begin{aligned}
& g_{\sigma}^{-2}\left(a_{\sigma}, \xi\right)=g^{-2}\left(a_{\sigma}\right)+c_{\sigma}(\xi)+\mathrm{O}\left(g^{2}\right), \\
& g_{\beta}^{-2}\left(a_{\sigma}, \xi\right)=g^{-2}\left(a_{\sigma}\right)+c_{\beta}(\xi)+\mathrm{O}\left(g^{2}\right)
\end{aligned}
$$

as the correct quantum coupling parameters in the continuum limit. Here $c_{\sigma}(\xi)$ and $c_{\beta}(\xi)$ are functions of $\xi$ only, vanishing at $\xi=1$.

As is known, the form (4.5) of the lattice action is not unique; at least in the $T=0$ case, other forms [23] lead to the same contınuum limit. The role of the choice of action for $T \neq 0$ is at present not known.

Expressing the derivatives with respect to the continuum variables $\beta$ and $V$ in terms of $\xi$ and $a_{\sigma}$, with $N_{\sigma}$ and $N_{\beta}$ fixed, we obtain

$$
\begin{aligned}
& \varepsilon_{\mathrm{E}}=\left(\xi^{2} / N_{\sigma}^{3} N_{\beta} a_{\sigma}^{4}\right)\left(\partial \ln Z_{\mathrm{E}} / \partial \xi\right)_{a_{\sigma}}, \\
& P_{\mathrm{E}}=\left(\xi^{2} / 3 N_{\sigma}^{3} N_{\beta} a_{\sigma}^{4}\right)\left[\left(\partial \ln Z_{\mathrm{E}} / \partial \xi\right)_{a_{\sigma}}+a_{\sigma} \xi^{-1}\left(\partial \ln Z_{\mathrm{E}} / \partial a_{\sigma}\right)_{\xi}\right],
\end{aligned}
$$

where we again use the subscript $E$ to remund ourselves that the vacuum correction needed in the euclidean form has not yet been performed. With the action (4.5), we have for $\mathrm{SU}(N)$ gauge fields

$$
\begin{aligned}
& \varepsilon_{\mathrm{E}} a_{\sigma}^{4}=-6 N \xi^{2}[\left.\left(\partial K_{\sigma} / \partial \xi\right) \bar{P}_{\sigma}+\left(\partial K_{\beta} / \partial \xi\right) \bar{P}_{\beta}\right], \\
& P_{\mathrm{E}} a_{\sigma}^{4}=-2 N \xi^{2}\left[\left(\partial K_{\sigma} / \partial \xi\right) \bar{P}_{\sigma}+\left(\partial K_{\beta} / \partial \xi\right) \bar{P}_{\beta}\right. \\
&\left.+a_{\sigma} \xi^{-1}\left\{\left(\partial K_{\sigma} / \partial a_{\sigma}\right) \bar{P}_{\sigma}+\left(\partial K_{\beta} / \partial a_{\sigma}\right) \bar{P}_{\beta}\right\}\right],
\end{aligned}
$$

where $\bar{P}_{\sigma}$ and $\bar{P}_{\beta}$ denote the average of space-like and space-temperature plaquettes, respectively:

$$
\begin{aligned}
\bar{P}_{\sigma} & \equiv Z_{\mathrm{E}}^{-1} \int \prod \mathrm{d} U_{l j} \mathrm{e}^{-S(U)}\left\{\sum_{\left\{\mathrm{P}_{\sigma}\right\}}\left[1-\frac{1}{N} \operatorname{Re} \operatorname{Tr} U U U U\right] / \sum_{\left\{\mathrm{P}_{\alpha}\right\}}\right\} \\
& =\left(3 N_{\sigma}^{3} N_{\beta} Z_{\mathrm{E}}\right)^{-1} \int \prod \mathrm{d} U_{t j} \mathrm{e}^{-S(U)} \sum_{\left\{\mathrm{P}_{o}\right\}}\left[1-\frac{1}{N} \operatorname{Re} \operatorname{Tr} U U U U\right],
\end{aligned}
$$

and correspondingly for $\bar{P}_{\beta}$. 
Using relations (4.11) and (4.12) with the $\mathrm{O}\left(g^{2}\right)$ corrections neglected, we obtain at $\xi=1$ from eqs. (4.15) and (4.16), with $a_{\mathrm{o}} \equiv a$,

$$
\begin{aligned}
& \varepsilon_{\mathrm{E}} a^{4}=6 N\left\{g^{-2}\left(\bar{P}_{\sigma}-\bar{P}_{\beta}\right)-\left(c_{\sigma}^{\prime} \bar{P}_{\sigma}+c_{\beta}^{\prime} \bar{P}_{\beta}\right)\right\}, \\
& P_{\mathrm{E}} a^{4}=2 N\left\{g^{-2}\left(\bar{P}_{\sigma}-\bar{P}_{\beta}\right)-\left(c_{\sigma}^{\prime} \bar{P}_{\sigma}+c_{\beta}^{\prime} \bar{P}_{\beta}\right)-a\left(\frac{\partial g^{-2}}{\partial a}\right)\left(\bar{P}_{\sigma}+\bar{P}_{\beta}\right)\right\} .
\end{aligned}
$$

To determine $\varepsilon$ and $P$ at $\xi=1$ as functions of the temperature $T=\left(N_{\beta} a\right)^{-1}$, we must therefore know $g(a)$ and the derivatives $c_{\sigma}^{\prime} \equiv\left(\mathrm{d} c_{\sigma} / \mathrm{d} \xi\right)_{\xi=1}$ and $c_{\beta}^{\prime} \equiv\left(\mathrm{d} c_{\beta} / \mathrm{d} \xi\right)_{\xi=1}$. In the continuum lımit, $g(a)$ is given by the renormalization group relation

$$
a \Lambda_{\mathrm{L}}=\exp \left\{-\frac{24 \pi^{2}}{11 N g^{2}}-\frac{51}{121} \ln \frac{11 N g^{2}}{48 \pi^{2}}\right\},
$$

obtained by requiring the invariance of physical observables, measured with $\Lambda_{L}$ as scale, under changes of the lattice spacing $a$. The derivatives $c_{\sigma}^{\prime}$ and $c_{\beta}^{\prime}$ can similarly be obtained by enforcing invariance under changes of $\xi$; one obtains, again in the continuum limit, for $\mathrm{SU}(N)$ the values [24]

$$
\begin{aligned}
& c_{\sigma}^{\prime}=4 N\left\{\frac{N^{2}-1}{32 N^{2}} 0.58684+0.00050\right\}, \\
& c_{\beta}^{\prime}=4 N\left\{-\frac{N^{2}-1}{32 N^{2}} 0.58684+0.00531\right\} .
\end{aligned}
$$

From eqs. (4.18) and (4.19) we obtain the quantity

$$
(\varepsilon-3 P)_{\mathrm{E}} a^{4}=6 N a\left(\partial g^{-2} / \partial a\right)\left(\bar{P}_{\sigma}+\bar{P}_{\beta}\right),
$$

which vanishes for an ideal gas and provides us with a measure of the interaction strength. In fact, if a perturbative treatment is valıd at high temperature, then we have [for SU(2)] to leading order [25]

$$
(\varepsilon-3 P) / T^{4}=0.611 \alpha_{\mathrm{s}}^{2}(T)-3.378 \alpha_{\mathrm{s}}^{5 / 2}(T) .
$$

Here $\alpha_{s}(T)=g^{2}(T) / 4 \pi$ denotes the effective strong interaction coupling constant at temperature $T$,

$$
\alpha_{\mathrm{s}}(T)=\frac{6 \pi}{11 N \ln (4 T / \Lambda)},
$$

with $\Lambda$ as continuum cut-off parameter. 
As mentioned, the euclidean form (4.4) still contains the vacuum contribution of $T=0$; we saw this explicitly in the cases studied in sects. 2 and 3 . Hence also now all expressions, $\varepsilon, P$, etc., must be adjusted by removing the $T=0$ value. On the asymmetric lattice of the finite temperature problem, this can be approximated by subtracting from any given quantity the corresponding form calculated on a suff1ciently large symmetric lattice. For the physical energy density $\varepsilon$ we thus get

$$
\varepsilon a^{4}=6 N\left\{g^{-2}\left(\bar{P}_{\sigma}-\bar{P}_{\beta}\right)+c_{\sigma}^{\prime}\left(\bar{P}-\bar{P}_{\sigma}\right)+c_{\beta}^{\prime}\left(\bar{P}-\bar{P}_{\beta}\right)\right\},
$$

since $\bar{P}_{\sigma}=\bar{P}_{\beta}=\bar{P}$ on a symmetric lattice. The physical form $P$ of the pressure is obtained correspondingly, and we have

$$
(\varepsilon-3 P) a^{4}=6 N a\left(\frac{\partial g^{-2}}{\partial a}\right)\left(\bar{P}_{\sigma}+\bar{P}_{\beta}-2 \bar{P}\right)
$$

for the interaction measure (4.22).

Finally we want to consider the free energy density of our system,

$$
f=\frac{-1}{\beta V} \ln Z .
$$

The Monte Carlo evaluation, as we shall see, permits only the calculation of lattice averages, with the weight $\exp (-S)$; hence eq. (4.27) is not in a suitable form for this method. We can, however, calculate

$$
-\left(\partial \ln Z_{\mathrm{E}} / \partial g^{-2}\right)_{\xi=1}=6 N N_{\sigma}^{3} N_{\beta}\left(\bar{P}_{\sigma}+\bar{P}_{\beta}\right)=g^{2} \bar{S},
$$

which is just the average action. As before, we normalize at $T=0$ by subtracting the corresponding value on a large symmetric lattice. Integrating over $g^{-2}$, we then obtain

$$
f a^{4}=6 N \int_{g_{0}^{-2}}^{g^{-2}} \mathrm{~d} \tilde{g}^{-2}\left[\bar{P}_{\sigma}+\bar{P}_{\beta}-2 \bar{P}\right]+c
$$

for the free energy density at the temperature

$$
T=\left(N_{\beta} a(g)\right)^{-1},
$$

with $a(g)$ given by relation (4.20); we therefore choose $g_{0}$ small enough to assure the validity of this relation. The integration constant $c$ is then given by

$$
c=f\left(T_{0}\right) a_{0}^{4},
$$

with $a_{0} \equiv a\left(g_{0}\right), T_{0}=\left(N_{\beta} a_{0}\right)^{-1}$. 


\section{The SU(2) Yang-Mills system at finite temperature}

In the last section we have obtained expressions for thermodynamic quantities formulated on a euclidean $N_{\sigma}^{3} \times N_{\beta}$ lattice, with spacings $a_{\sigma}, a_{\beta}$ and associated coupling parameters $g_{\sigma}, g_{\beta}$. We would like to evaluate these expressions in the thermodynamic limit $\left(V=\left(N_{\sigma} a_{\sigma}\right)^{3} \rightarrow \infty\right)$ at temperature $T=\left(N_{\beta} a_{\beta}\right)^{-1}$, in a region of lattice spacings sufficiently small to render our results independent of the lattice structure (continuum limit: $a_{\sigma}, a_{\beta} \rightarrow 0$ ). While it is possible to carry out analytic calculations in perturbation expansions with $g^{2} \rightarrow \infty$ [3] or $g^{2} \rightarrow 0$ [26], the only way to cover the entire temperature range is so far given by Monte Carlo simulation.

In confınement studies, e.g. for the string tension $\sigma$, the Monte Carlo evaluation has led to two distinct regions: a strong coupling regime, in which the numerical results follow the lowest order of the $g^{2} \rightarrow \infty$ expansion, and a weak coupling regime, $g^{2} \rightarrow 0$, in which the renormalization relation (4.20) is satısfied. Only from this weak coupling region it is possible to extract the continuum values of physical quantities. We note here that also finite temperature thermodynamics can for all $T$ be considered as a weak coupling problem: by choosing a sufficiently large $N_{\beta}$ at fixed $T=\left(N_{\beta} a_{\beta}\right)^{-1}, a_{\beta}$ and hence the associated coupling can be made as small as desired. In fact, only in the region of validity of eq. (4.20) does it appear possible to connect a lattice formulation with given coupling $g$ unambıguously to a specific physical temperature.

The actual Monte Carlo evaluation is now carried out as follows. The computer simulates an $N_{\sigma}^{3} \times N_{\beta}$ lattice; for convenience we choose again $\xi=1\left(a_{\sigma}=a_{\beta}=a\right)$. With $c_{\sigma}^{\prime}$ and $c_{\beta}^{\prime}$ as given by eq. (4.21) all expressions are functions of $g$, and hence in the region of validity of eq. (4.20) of the physical temperature $T=\left(N_{\beta} a(g)\right)^{-1}$. For a given $g$, starting from some specified configuration of group elements $U$ on the lattıce, each lınk is assigned a new $U^{\prime}$, chosen randomly with weight $\exp [-S(U)]$. The starting configuration is etther taken as completely ordered (all $U=1$, "cold start") or as completely disordered (all $U$ random, "hot start"). One passage of this procedure through the entire lattice is called an iteration, and after a thousand or more iterations results tend to become quite stable even on farrly small lattices. To obtain some intuitive feeling for this, note that a $10^{3} \times 3$ lattice has 12000 link degrees of freedom, thus providing us with a rather large statıstıcal system

The calculations presented here are generally performed on a $10^{3} \times 2,3,4,5$ lattice; for $N_{\beta}=3$, we have typically 5000 iterations per point, using the icosahedral subgroup of $\mathrm{SU}(2)$, which we found to agree with corresponding calculations using the full group. The errors shown are generally twice the statistical errors.

\section{HIGH-TEMPERATURE BEHAVIOUR}

At sufficiently high temperatures, we expect Yang-Mılls systems to behave like a photon gas with the appropriate number of degrees of freedom. For a massless $\mathrm{SU}(2)$ vector field, with two spin and three gluonic colour degrees of freedom, the 
resulting Stefan-Boltzmann form is

$$
\varepsilon_{\mathrm{SB}}=3 \frac{\pi^{2}}{15} T^{4}=\frac{\pi^{2}}{5} T^{4}
$$

Together with eq. (4.20), this predicts on the lattıce

$$
\varepsilon_{\mathrm{SB}} a^{4}=\frac{\pi^{2}}{5}\left(\frac{T}{\Lambda_{\mathrm{L}}}\right)^{4} \exp \left(-4\left\{\frac{12 \pi^{2}}{11 g^{2}}+\frac{51}{121} \ln \frac{11 g^{2}}{24 \pi^{2}}\right\}\right),
$$

for the dimensionless quantity $\varepsilon a^{4}$ at fixed $T$ as function of $g^{2}$. In fig. 7, we compare eq. (5.2) with the results obtained from eq. (4.25) by Monte Carlo evaluation, at $T=500 \Lambda_{\mathrm{L}}, N_{\sigma}=10$, and $N_{\beta}=2,3,4,5$.

In comparing eq. (4.25) and (5.2), we note that the functional behaviour in $g^{2}$ tests the validity of the renormalization relation, the absolute normalization that of the Stefan-Boltzmann factor $\frac{1}{5} \pi^{2}-1 . e$., it provides direct evidence for the colour degrees

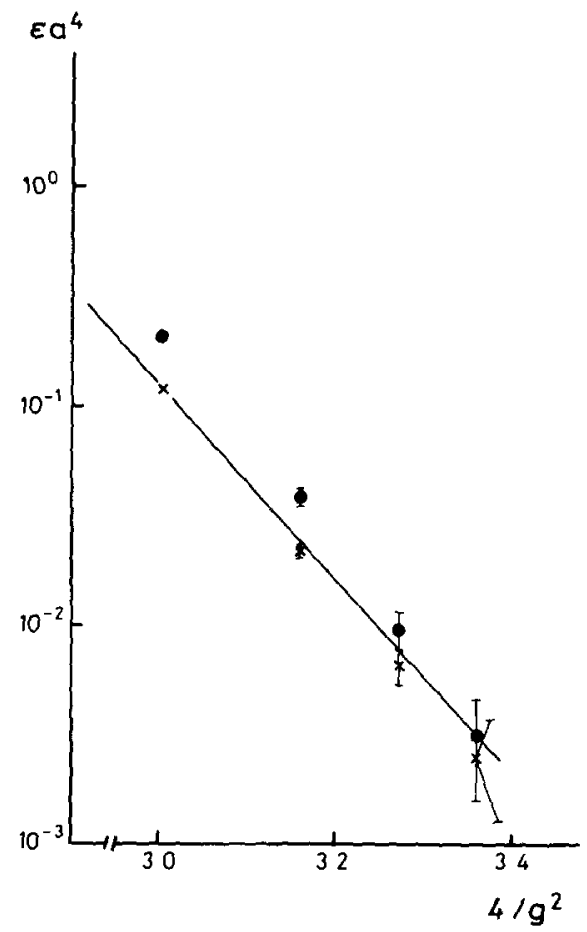

Fig 7 Energy density of the SU(2) Yang-Mills system versus $4 / g^{2}$, at fixed temperature $T=500 \mathrm{\Lambda}_{L}$, after about 600 iterations (full points) The sold line gives the Stefan-Boltzmann limit, the crosses are the finite size corrected results 


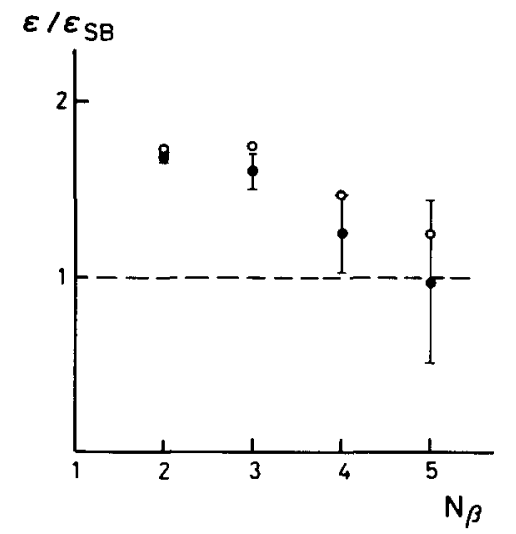

F1g 8 The uncorrected ratio $\varepsilon / \varepsilon_{\mathrm{SB}}$ at $T=500 \Lambda_{\mathrm{L}}$ versus $N_{\beta}$ for the $\mathrm{SU}(2)$ Yang-Mills system (full points) and for the ideal Bose gas (circles)

of freedom operative in the system. We see in fig. 7 that $\varepsilon a^{4}$ on the whole follows the asymptotic form (5.2), but there appear to be stronger deviations at smaller $N_{\beta}$. In f1g. 8 we compare these with the deviations observed for an 1deal Bose gas on a lattice of the same size (see fig. 6). Both coincide, supporting strongly the supposition that the deviations found for the $\mathrm{SU}(2)$ system are also predominantly finite lattice effects. We have therefore multiplied the results of fig. 7 by the correction factor $C\left(N_{\sigma}, N_{\beta}\right) \equiv \varepsilon_{\mathrm{SB}} / \varepsilon$ from the 1deal Bose gas [21]. These corrected results are also shown in fig. 7 ; they are seen to be $N_{\beta}$ independent, and within errors they agree with the asymptotic Stefan-Boltzmann form. Unless mentioned, we shall therefore use from now on only corrected values.

At $T=500 \Lambda_{\mathrm{L}}$, the energy density effectively becomes the difference of plaquette averages,

$$
\varepsilon a^{4} \simeq 12 g^{-2}\left(\bar{P}_{\sigma}-\bar{P}_{\beta}\right)
$$

the other two terms contribute less than fifteen percent ${ }^{\star}$. Such a behaviour is asymptotically expected, since all plaquette averages vanish as $g^{2}$ in the weak coupling expansion on the lattice [26], and only the first term has a compensating $g^{-2}$. This first term, for $N_{\beta}=3$, in fact is found to agree with the Stefan-Boltzmann form (5.1) down to $T \simeq 90 \Lambda_{\mathrm{L}}$.

In fig. 9, we show the high-temperature behaviour of the interaction measure (4.26), calculated on $10^{3} \times 3$ and $10^{3} \times 2$ lattices, using relation (4.20) and for $\bar{P}$ the results of ref. [27]. We see that for $T \gtrsim 200 \Lambda_{\mathrm{L}}$, our results are compatible with an asymptotically free gluon gas. Also shown in fig. 9 is the perturbation form (4.23),

\footnotetext{
* In earlier work [7], we estımated a still smaller contribution from these two terms, because it was there assumed that $g_{\sigma}=g_{\beta}$
} 


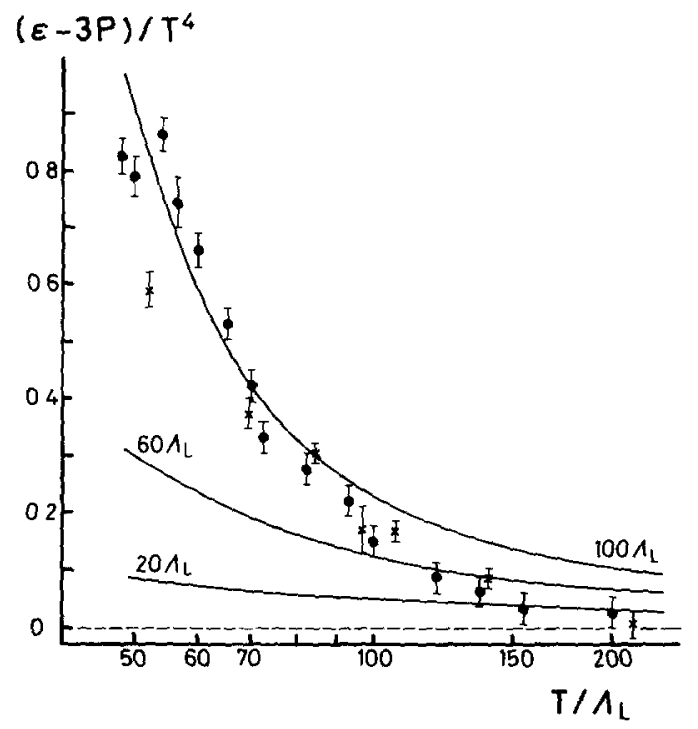

Fig. 9 The difference $(\varepsilon-3 P) / T^{4}$ versus temperature for $N_{o}=10, N_{\beta}=2$ (crosses) and $N_{\beta}=3$ (full points) The curves show the predictions of the first-order perturbation theory [ $\alpha_{s}^{2}$ term in eq $\left.(423)\right]$, the numbers on the curves are the corresponding continuum scale parameter values $\Lambda$

keeping only the $\alpha_{\mathrm{s}}^{2}(T)$ term with

$$
\alpha_{\mathrm{s}}(T)=3 \pi /(11 \ln (4 T / \Lambda)) .
$$

The deviations from ideal gas behaviour thus appear to be compatible in size with such a correction, if $\Lambda \simeq(20-100) \Lambda_{L}$. Note, however, that the next term $\left(\alpha_{\mathrm{s}}^{5 / 2}\right)$ in the expansion (4.23) would with this value of $\Lambda$ give a larger contribution than the $\boldsymbol{\alpha}_{\mathrm{s}}^{2}$ term, throwing considerable doubt on the validity of a perturbative treatment with a finite number of terms in the temperature region treated here.

Finally we consıder the negative of the free energy density (4.29), which for an asymptotically free gas should approach $\frac{1}{3} \varepsilon$ in the same way as the pressure. Integrating from $4 / \mathrm{g}^{2}=1.7$ up to 2.8 on a $10^{3} \times 2$ lattice, and from $4 / \mathrm{g}^{2}=1.8$ up to 2.8 on a $10^{3} \times 3$ lattice, with $\bar{P}$ again from ref. [27] we obtain the (corrected) values

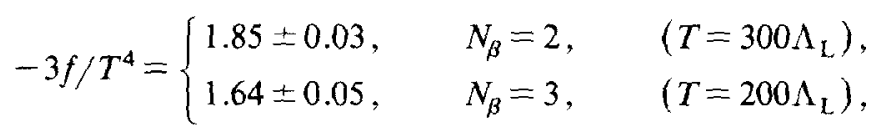

to be compared with

$$
\varepsilon / T^{4}= \begin{cases}1.93 \pm 0.01, & N_{\beta}=2, \\ 177 \pm 0.03, & N_{\beta}=3,\end{cases}
$$


at the corresponding temperatures; recall that $\varepsilon_{\mathrm{SB}} / T^{4}=\frac{1}{5} \pi^{2}=1.97$. The values (5.6) are for given $N_{\beta}$ obtained at one value of $\mathrm{g}^{2}$, and determined mainly by the difference of space-like and temperature-like plaquette averages In contrast, the values (5.5) are obtained by integratıng a rapidly varyıng function $\left(\sim(\varepsilon-3 P) / T^{4}\right)$ over a range of $g^{2}$ (or equivalently, of temperature), including the critical region; moreover, they depend on the sum of space-like and temperature-like plaquette averages, with the vacuum contribution subtracted. It seems remarkable that the results of such different evaluation procedures agree so well.

Combining the results for $\varepsilon, \varepsilon-3 P$ and $f$, we then conclude that at high temperatures, $T \gtrsim(100-200) \Lambda_{\mathrm{L}}$, the $\mathrm{SU}(2)$ Yang-Mills system approaches the asymptotic 1 deal gas form. As final illustration for this, we show in fig. 10 the high temperature behaviour of $\varepsilon / \varepsilon_{\mathrm{SB}}$, as calculated on $10^{3} \times 2$ and $10^{3} \times 3$ lattices, with finite size corrections.

Before going on to lower temperatures, let us comment on the physical scale for the region just considered. Bearing in mind the reservations necessary when empir1cal data is combined with an SU(2) Yang-Mills description, we can use the string tension value $[4,28] \Lambda_{\mathrm{L}} \simeq 5 \mathrm{MeV}$ to conclude that our high temperature region 100 to $500 \Lambda_{\mathrm{L}}$ corresponds to temperatures $T \simeq 500-2500 \mathrm{MeV}$; we are thus more than a factor 2 above the canonical "critical hadron temperature" [1] of $150-200 \mathrm{MeV}$.

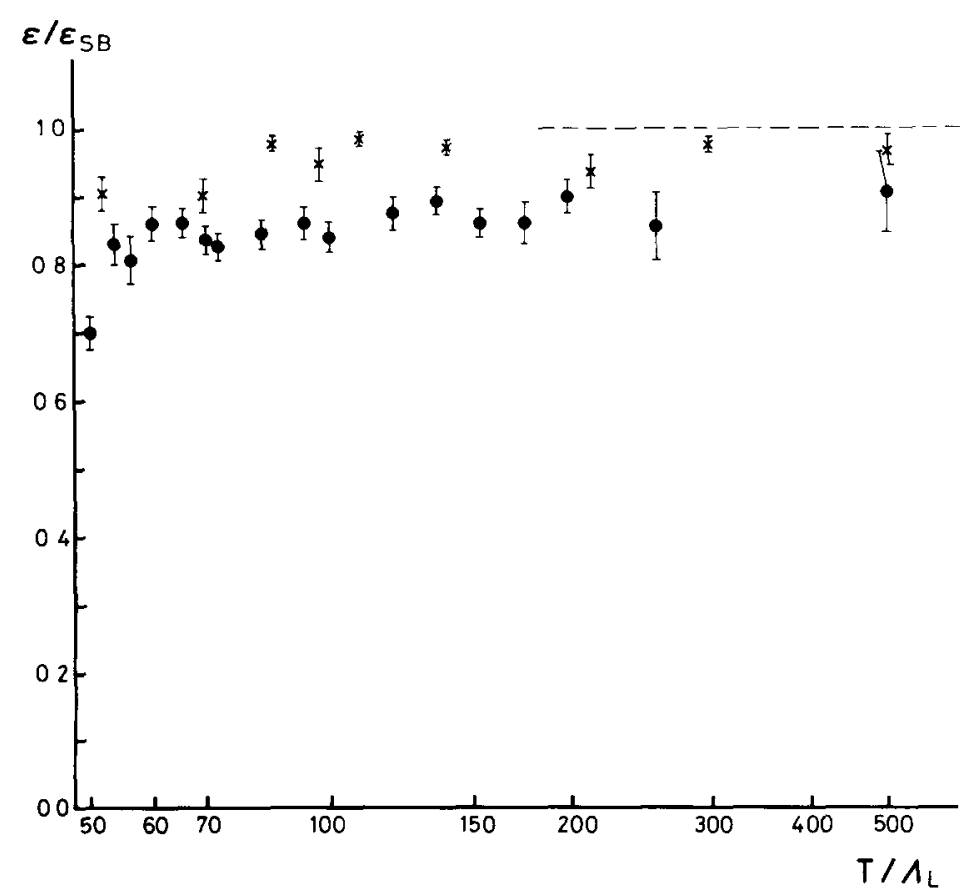

Flg 10 High-temperature behaviour of $\varepsilon / \varepsilon_{\mathrm{SB}}$ versus temperature, as calculated on $10^{3} \times 2$ (crosses) and $10^{3} \times 3$ (full points) lattıces 


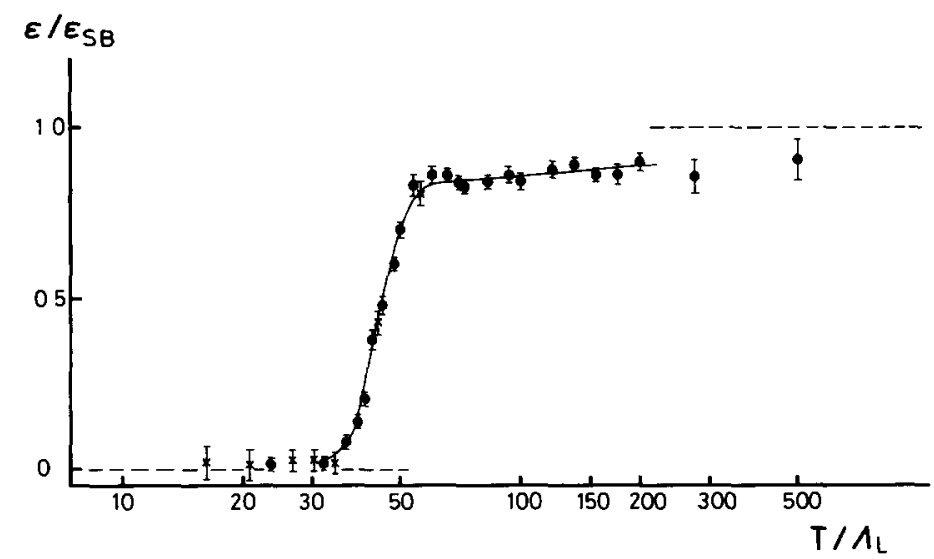

Fig 11 The ratio $\varepsilon / \varepsilon_{\mathrm{SB}}$ for a $10^{3} \times 3$ lattice versus temperature The full points are icosahedral. the crosses full SU(2) group Monte Carlo results; the line is a spline fit to the data

\section{THE DECONFINEMENT TRANSITION}

In fig. 11 , we now display the behaviour of $\varepsilon / \varepsilon_{\mathrm{SB}}$ over the entire temperature range accessible to us within reasonable computer time. The most striking feature is the sharp drop near $T=40 \Lambda_{\mathrm{L}}$, where the energy density falls an order of magnitude in a temperature interval of about $5 \Lambda_{\mathrm{L}}(\sim 25 \mathrm{MeV})$, thus displaying a behaviour very similar to that of the gauge-invariant Ising model. The behaviour of the specific heat per unit volume,

$$
c_{\mathrm{V}}=\partial \varepsilon / \partial T
$$

obtained by differentiation of a fit to the data of fig. 1l, is shown in fig. 12. It exhibits a strong singularity like peak at $T_{\mathrm{c}}=43 \Lambda_{\mathrm{L}}$, which we take as signal of the deconfinement transition.

We recall that the evaluation of the SU(2) Yang-Mills system on a given lattice is performed at fixed $g^{2}$; the connection to the temperature is then provided by the renormalization group relation (4.20). To assure that the deconfinement signal occurs indeed at fixed $T$, we must therefore observe a shift in the peak of $c_{\mathrm{V}}$ as function of $g^{2}$. In fig. 13 , we show $\left[\partial\left(\varepsilon / \varepsilon_{\mathrm{SB}}\right) / \partial\left(4 / g^{2}\right)\right]$ versus $4 / g^{2}$ as calculated on $10^{3} \times 2$ and $10^{3} \times 3$ lattices. For $N_{\beta}=2$, the peak occurs at $4 / \mathrm{g}^{2}=1.9$, while for $N_{\beta}=3$, it is shifted to $4 / g^{2}=2.19$. Moreover, the peak becomes sharper and higher with increasing $N_{\beta}$. For $N_{\beta}=4$, the deconfinement signal occurs at $4 / g^{2} \simeq 2.3$. Using the renormalization relation (4.20), both the $N_{\beta}=3$ and the $N_{\beta}=4$ results give $T_{\mathrm{c}}=43 \Lambda_{\mathrm{L}}$; the $N_{\beta}=2$ position is in accordance with this, if instead of eq. (4.20) we use the non-asymptotic string-tension results [4] to connect $g^{2}$ and $a$. 


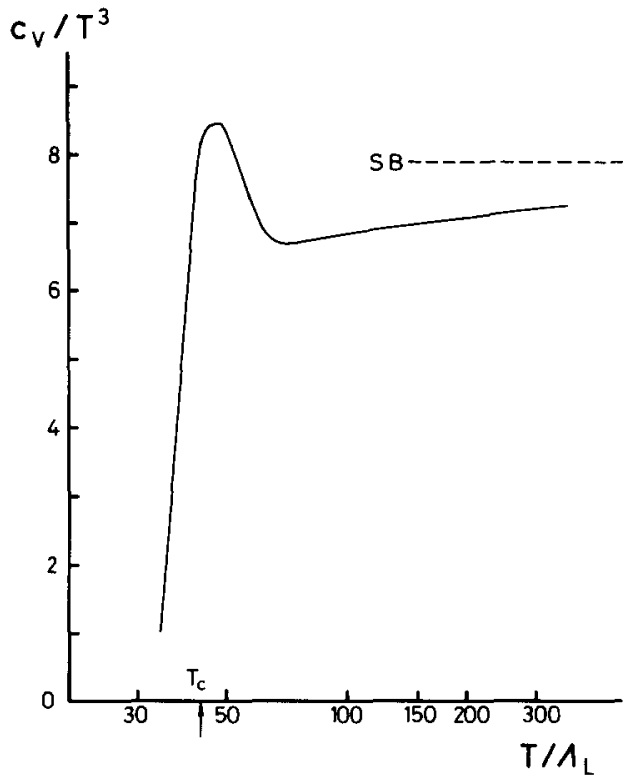

Fig 12 The specific heat per unit volume divided by $T^{3}$ versus temperature, obtaned from the fit in fig 11 to $\varepsilon / \varepsilon_{\mathrm{SB}}$

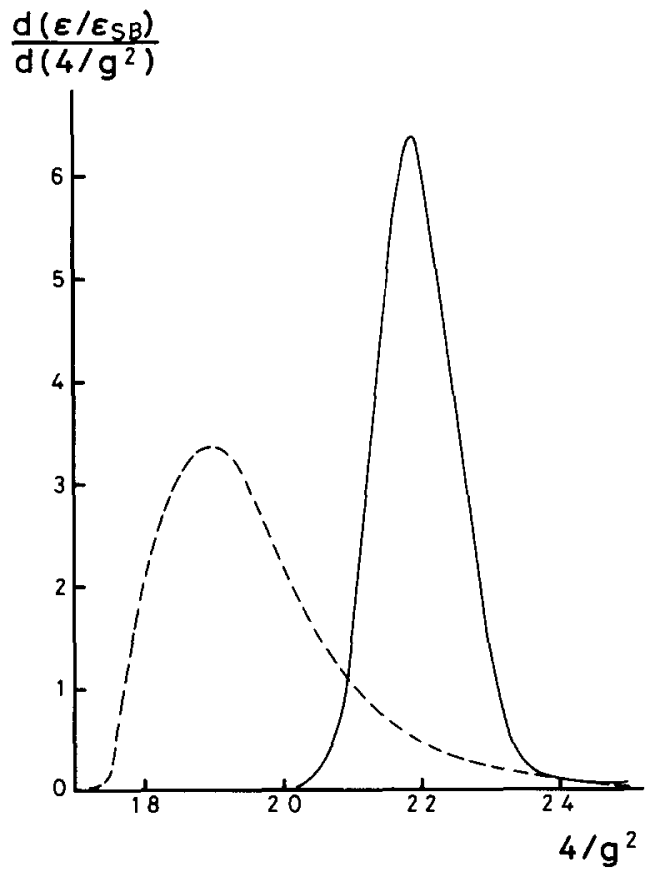

Fig 13 The denvative $\mathrm{d}\left(\varepsilon / \varepsilon_{\mathrm{SB}}\right) / \mathrm{d}\left(4 / \mathrm{g}^{2}\right)$ versus $4 / \mathrm{g}^{2}$ for $N_{\mathrm{o}}=10, N_{\beta}=2$ (dashed line) and $N_{\beta}=3$ (solid line), obtained from spline fits in $4 / g^{2}$ to the ratios $\varepsilon / \varepsilon_{S B}$ 


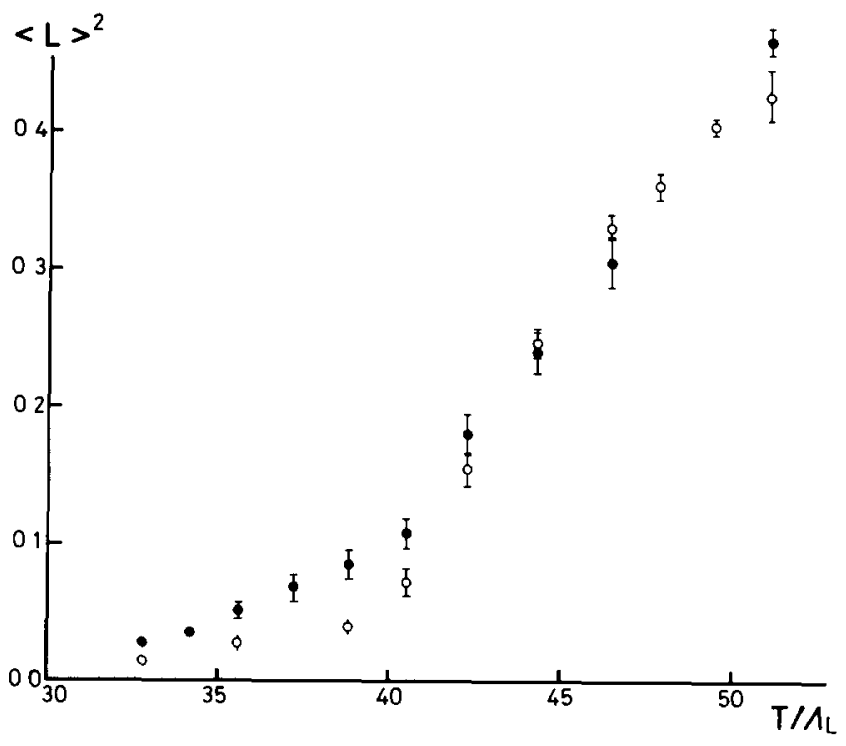

Fig 14 Thermal Wilson loop $\langle L\rangle^{2}$ versus temperature for $N_{\beta}=3, N_{\sigma}=7$ (full points) and $N_{\sigma}=9$ (circles)

To further support the claim that the peak we observe in the specific heat is a signal of deconfinement, we have also here calculated the square of the average* thermal Wilson loop $\langle L\rangle^{2}$. As already mentioned in sect. $2, \ln |\langle L\rangle|$ measures the free energy $F$ of an isolated colour charge and can be used as an order parameter for gauge-invariant systems. In the confinement region, $F$ should be infinite, forcing $|\langle L\rangle|$ to vanish. In fig. 14 , we find in accordance with the results of refs. $[5,6]$ that there is indeed an abrupt increase of $\langle L\rangle^{2}$ at about $42 \Lambda_{\mathrm{L}}$. We note, moreover, that with increasing spatial lattice size $\left(N_{\sigma}=7 \rightarrow N_{\sigma}=9\right)$, the values of $\langle L\rangle^{2}$ below $T_{\mathrm{c}}$ move closer to zero, as expected if $\langle L\rangle^{2}=0$ for $N_{\sigma} \rightarrow \infty$.

Before looking in more detail at the "hadronic" region below $T_{\mathrm{c}}$, we note that the critical energy density, $\varepsilon_{\mathrm{c}} \equiv \varepsilon\left(T_{\mathrm{c}}\right)$, is obtained here as $\varepsilon_{\mathrm{c}} \simeq T_{\mathrm{c}}^{4}$ (see fig. 11). With $\Lambda_{\mathrm{L}}=5 \mathrm{MeV}$, we have thus $\varepsilon_{\mathrm{c}} \simeq 270 \mathrm{MeV} / \mathrm{fm}^{3}$. To get some feeling for this value, recall that the energy density in nuclear matter is about $160 \mathrm{MeV} / \mathrm{fm}^{3}$, while inside a nucleon it is $360 \mathrm{MeV} / \mathrm{fm}^{3}$.

In fig. 15 we now consider the interaction measure $(\varepsilon-3 P) / T^{4}$ over the entire temperature range. The interaction vanıshes at high temperatures, leaving us, as we saw, with an asymptotically free gluon gas. We note that at low temperatures it also vanishes, suggestıng that we there have an ideal gas of "hadron-like" objects, which in a pure Yang-Mills theory must be gluonium states.

* Because of the exact $Z_{2}$ symmetry of the problem, $\langle L\rangle^{2}$ is a convenuent quantity to consider It is calculated by squaring the average of $L$ over the lattice after each iteration 


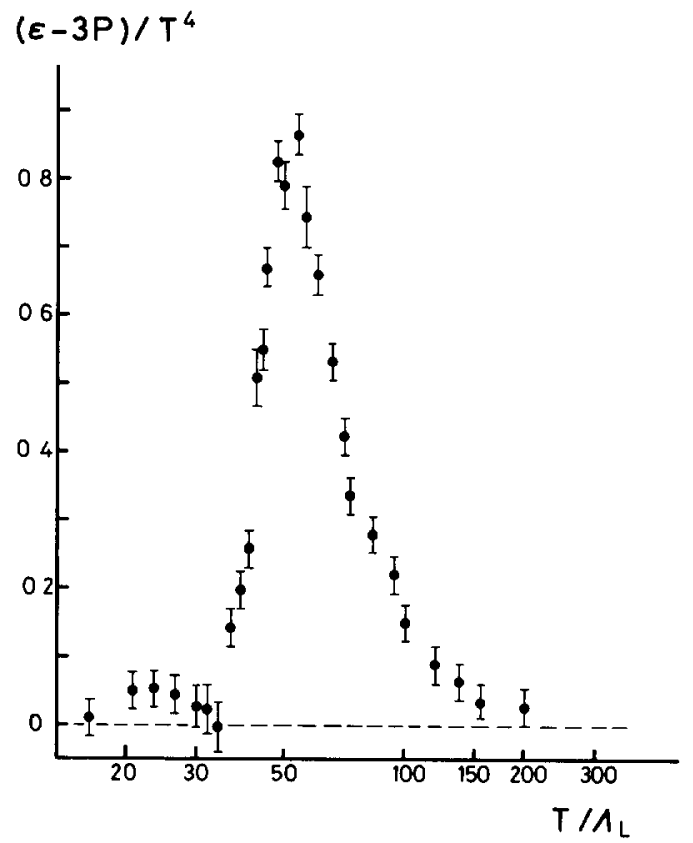

Fig 15 The difference $(\varepsilon-3 P) / T^{4}$ versus temperature for a $10^{3} \times 3$ lattice

\section{THE GLUONIUM REGION}

Below the deconfinement temperature, strongly interacting matter should consist of hadrons in interaction. For a pure Yang-Mills system, in the absence of quarks, this implies a gas whose constituents are bound gluon states, 1.e., gluonia. We therefore want to compare the results of our lattice evaluation in the region below $T_{\mathrm{c}}$ with the continuum behaviour expected for a system of interacting gluonium states. To have a defınite form for the behaviour of such a system, we assume it to follow the pattern of hadronic matter, which is generally taken as an ideal gas of the ground-state hadrons and all their resonance excitations.

The partition function for an ideal gas of resonances is given by

$$
\frac{1}{V} \ln Z(T, V)=(2 \pi)^{-3} \int_{0}^{\infty} \mathrm{d} m \tau(m) \int \mathrm{d}^{3} p \mathrm{e}^{-\sqrt{p^{2}+m^{2}} / T},
$$

where $\tau(m)$ denotes the excitation spectrum. From dual [29] or bag [30] models as well as from statistical bootstrap arguments [31], we expect $\tau(m)$ to have the form

$$
\tau(m)=d \delta\left(m-m_{0}\right)+c \theta\left(m-2 m_{0}\right) m^{-a} \mathrm{e}^{b m},
$$

with constant $a, b, c$ and $d$. While $a$ depends on the details of the model [32], $b$ is 
fixed by the bag pressure or string tension (Regge slope), and $c$ measures the strength of resonance excitation relative to the $d$-fold degenerate ground state.

It is well known that the partition function (5.8) with the spectrum (5.9) leads to critical behaviour [33], with $T_{c}=1 / b$ as critical temperature. The order of the associated transition is determined by $a$; in particular, for $\frac{7}{2}<a \leqslant \frac{9}{2}$, we have a finite $\varepsilon\left(T_{\mathrm{c}}\right)$, while $c_{\mathrm{V}}$ diverges as $T \rightarrow T_{\mathrm{c}}$. Assuming that the critical behaviour of such a system can be identified with what we find in lattice QCD, we shall therefore fix $b^{-1}=43 \Lambda_{\mathrm{L}}$ and maintain $a$ in the mentioned range. We furthermore take $d=6$, in accord with bag model [35] and strong coupling arguments [34]. The remaining constant $c$ is fixed by normalizing resonance gas results to our Monte Carlo value of $\varepsilon\left(T_{c}\right)$. This leaves us as only open parameter of the resonance gas description the value $m_{0}$ of the lowest hadronic state - here the mass of the glueball, $m_{0}=m_{\mathrm{G}}$.

From eqs. (5.8) and (5.9) we have

$$
\varepsilon(T)=\varepsilon_{\mathrm{G}}(T)+\frac{c T}{2 \pi^{2}} \int_{2 m_{\mathrm{G}}}^{\infty} \mathrm{d} m m^{3-a} \mathrm{e}^{m b}\left[K_{1}\left(\frac{m}{T}\right)+\frac{3 T}{m} K_{2}\left(\frac{m}{T}\right)\right],
$$

where

$$
\varepsilon_{\mathrm{G}}(T)=d \frac{m_{\mathrm{G}}^{3} T}{2 \pi^{2}}\left[K_{1}\left(\frac{m_{\mathrm{G}}}{T}\right)+\frac{3 T}{m_{\mathrm{G}}} K_{2}\left(\frac{m_{\mathrm{G}}}{T}\right)\right]
$$

denotes the energy density of an 1deal glueball gas (w1thout resonances). The corresponding specific heat per unit volume is given by

$$
\begin{aligned}
c_{\mathrm{V}}(T)=\frac{1}{T}\{ & \varepsilon(T)+\frac{1}{2 \pi^{2}} \int_{2 m_{\mathrm{G}}}^{\infty} \mathrm{d} m m^{4} \tau(m) \\
& \left.\times\left[K_{2}\left(\frac{m}{T}\right)+\frac{2 T}{m} K_{1}\left(\frac{m}{T}\right)+9\left(\frac{T}{m}\right)^{2} K_{2}\left(\frac{m}{T}\right)\right]\right\},
\end{aligned}
$$

and for the interaction measure (4.26) we obtain

$$
(\varepsilon-3 P) / T^{4}=\frac{1}{2 \pi^{2} T^{3}}\left\{d m_{\mathrm{G}}^{3} K_{1}\left(\frac{m_{G}}{T}\right)+c \int_{2 m_{\mathrm{G}}}^{\infty} \mathrm{d} m m^{3-a} \mathrm{e}^{m b} K_{1}\left(\frac{m}{T}\right)\right\} .
$$

With all parameters chosen as discussed above, we can now compare the gluonium gas forms (5.10), (5.12) and (5.13), which incorporate our expectations for hadronic matter, with the lattice results for the $\mathrm{SU}(2)$ Yang-Mills system.

In figs. 16 and 17 , we show $\varepsilon / T^{4}$, normalized to its value at $T_{\mathrm{c}}$, as a function of $x=T_{\mathrm{c}} / T-1$, comparing eq. (5.10) with the results from the SU(2) Yang-Mills system on a $10^{3} \times 3$ lattice. The dependence on the precise value of $a$ within the 


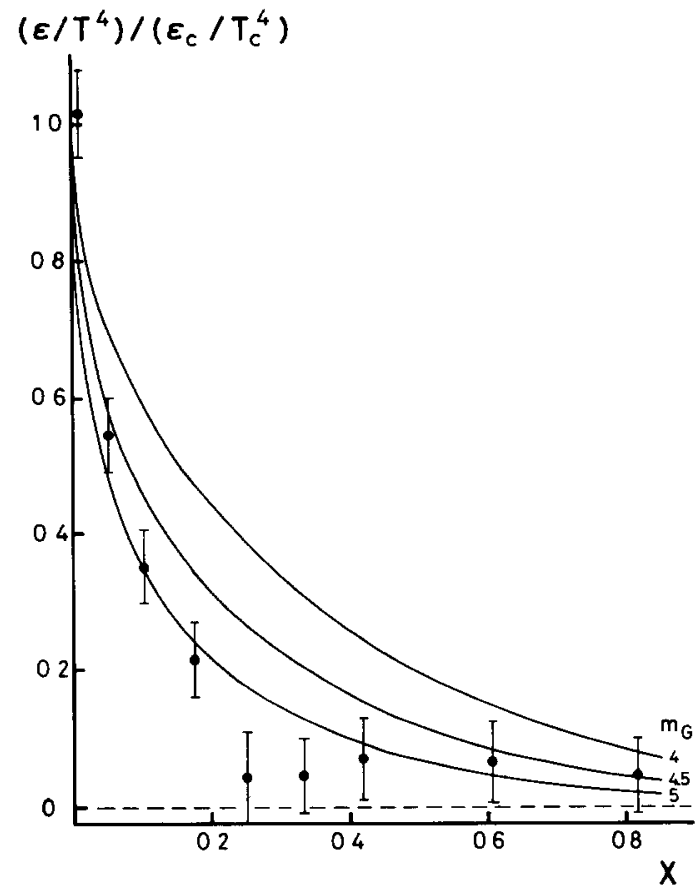

Fig 16 The energy density divided by $T^{4}$ and normalized at the critical temperature versus the variable $\mathrm{r}=T_{\mathrm{c}} / T-1$ The curves are the resonance gas predictions for $a=4$ and $m_{\mathrm{G}}=4,45$ and 5 times $T_{\mathrm{c}}$

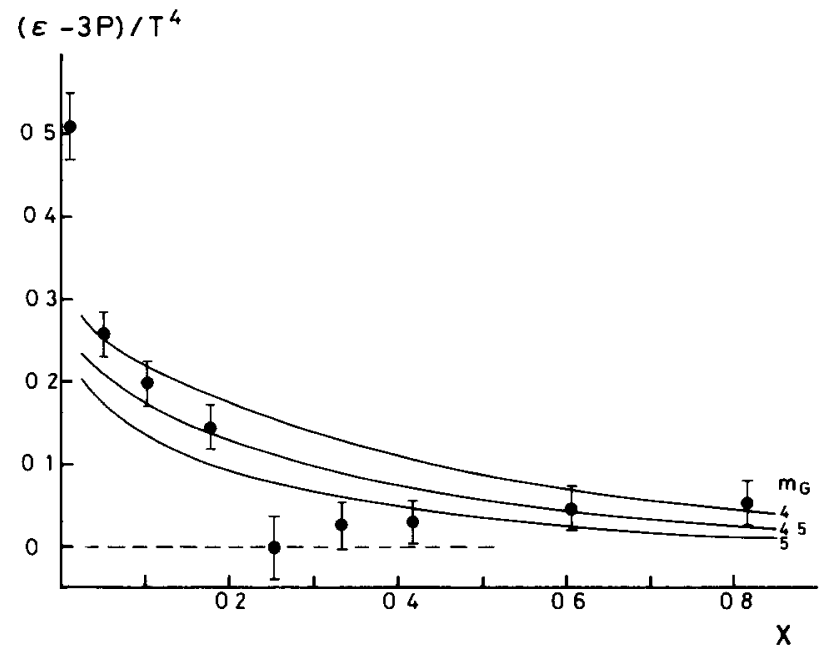

F1g 17 The difference $(\varepsilon-3 P) / T^{4}$ versus $x$ Notations and predictions correspond to that of $f 1 g 16$ 


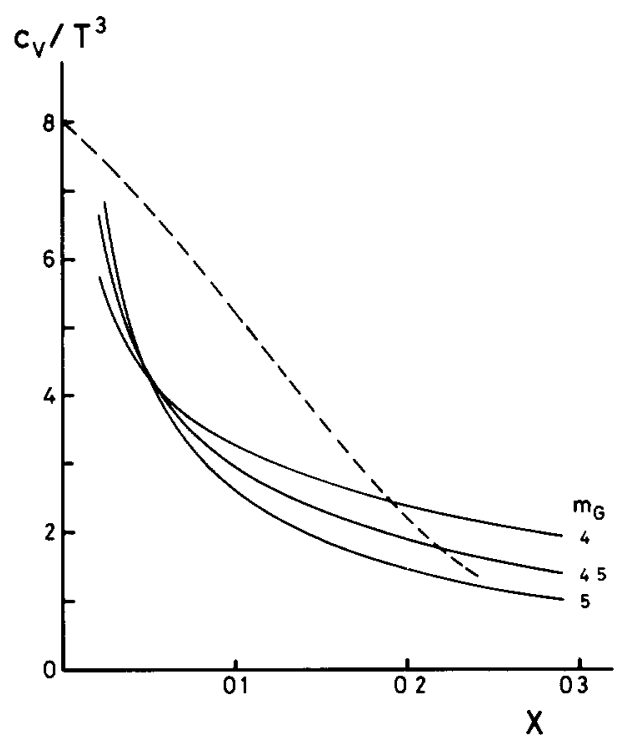

Fig 18 The specific heat per unit volume divided by $T^{3}$ versus $x$ The dashed line corresponds to the low temperature part of fig 12 , the solid lines to the resonance gas predictions as in figs 16 and 17

range $\frac{7}{2}<a \leqslant \frac{9}{2}$ is rather insignificant. In fig. 16 the results for different values of the glueball mass $m_{\mathrm{G}}$ are compared, with $a=4$. We see that $m_{\mathrm{G}}=(4-5) T_{\mathrm{c}}$ gives a quite reasonable fit.

In fig. 17, the corresponding results are shown for the interaction measure $(\varepsilon-3 P) / T^{4}$, and in fig. 18 for $c_{\mathrm{V}} / T^{3}$. Again the gluonium gas forms, with $m_{\mathrm{G}}=(4-5) T_{\mathrm{c}}$, are in reasonable agreement with the results of the Yang-Mills system. The fairly large discrepancies in $c_{\mathrm{V}} / T^{3}$ at small $x$ are presumably finite size effects; the resonance gas $c_{\mathrm{V}}$ diverges at $x=0$, which the lattice form could reproduce only in the continuum limit.

From the qualitative agreement and the quantitative comparisons in figs. 16-18 we thus conclude that low-temperature SU(2) Yang-Mills thermodynamics leads to a glueball mass of $m_{\mathrm{G}}=(200 \pm 50) \Lambda_{\mathrm{L}}$. In terms of the string tension $\sigma$, with the relation [4] $\Lambda_{\mathrm{L}}=(0.013 \pm 0.002) \sqrt{\sigma}$, we have $m_{\mathrm{G}}=(2.6 \pm 0.5) \sqrt{\sigma}$, in fair agreement with other glueball mass determinations from SU(2) Yang-Mills studies on the lattice $[28,36]$. In terms of physical dimensions, with $\Lambda_{\mathrm{L}} \approx 5 \mathrm{MeV}$, we obtain $m_{\mathrm{G}}=1000 \pm 200 \mathrm{MeV}$. These results, though compatible with earlier work on a smaller lattice [10], seem to lie systematically higher than what was found there; this in part due to the approximation mentioned in the footnote in subsect 5.1 , in part it could also be a finite size effect.

If we picture strongly interacting matter below $T_{c}$ as a gas of bound gluon states, which will have a dimension, it appears natural to ask whether the spatial lattice on our Monte Carlo evaluation is in fact large enough to accommodate a large number 
of such extended objects. The lattice spacing at $T_{\mathrm{c}} 1 \mathrm{~s}$, on a lattıce with $N_{\beta}=3$,

$$
a_{\mathrm{c}} \simeq 7.75 \times 10^{-3} \Lambda_{\mathrm{L}}^{-1},
$$

which with $\Lambda_{\mathrm{L}}=5 \mathrm{MeV}$ becomes about one-third of the nucleonic charge radius:

$$
a_{\mathrm{c}} \simeq 03 \mathrm{fm} .
$$

The spatial volume of our $10^{3}$ lattice at $T_{\mathrm{c}}$ is thus about $37 \mathrm{fm}^{3}$, or 60 tımes the volume of a nucleon. With such parameter values, a resonance gas description does not seem unreasonable. For lower temperatures, the spatial volume is increased by a factor $\left(T_{\mathrm{c}} / T\right)^{3}$.

\section{Conclusions and outlook}

We have found that the SU(2) Yang-Mills system, evaluated in the euclidean lattice formulation, provides a unified thermodynamic description of strongly interacting matter, predicting with increasing temperature a deconfinement transition from the "hadronic" gluonium phase to the asymptotically free gluon phase.

The gluonium phase shows the behaviour of a resonance gas, with a discrete ground state ("glueball") of mass $m_{\mathrm{G}} \simeq 200 \Lambda_{\mathrm{L}}$. The deconfinement transition, signalled by a singularity in the specific heat, occurs at $T_{\mathrm{c}} \simeq 43 \Lambda_{\mathrm{L}}$. At high temperature $\left(T \gtrsim 200 \Lambda_{\mathrm{L}}\right)$, the energy density approaches the Stefan-Boltzmann form for gluons with three colour degrees of freedom [for SU(2)].

Our calculations were generally performed on lattices with $10^{3}$ spatial sites and $2-4$ sites on the temperature axıs. Concerning the dependence on lattice size, we find at high temperatures the same deviations from Stefan-Boltzmann behaviour as are observed for an ideal Bose gas on a corresponding lattice. Further lattice size studies, in particular tests of finite size scalıng for the deconfinement singularity, would certainly be of interest and seem computationally feasible, but lengthy.

The approach to asymptotically free behaviour at high temperature is, in our results, not compatible with conventional perturbation theory up to and including third order. Since asymptotic freedom could be "non-perturbative", an expansion in $g^{2}$ need not converge, and difficulties arısing from infrared-divergent terms have in fact been discussed [37].

A problem of special interest is of course the extension of our considerations to a theory with fermions - to investigate whether the introduction of quarks leads to any qualitative or quantitative changes. A particularly intriguing question here is the occurrence of more than one transition - as argued by percolation studies [38] or in connection to chiral symmetry restoration [39].

It is a pleasure to thank P. Hasenfratz, L. McLerran and B. Petersson for useful discussions in various stages of thıs work. 


\section{References}

[1] Hadronic matter at extreme energy density, ed N Cabibbo and L Sertono (Plenum, New York, London, 1978)

[2] E V Shuryak, Phys Reports 61 (1980) 71,

D J Gross, R D Pisarsk1, and L G Yaffe, Rev Mod Phys 53 (1981) 43,

H Satz, Proc 5th High energy heavy ion study, Berkeley/Cal, 1981

[3] A M Polyakov, Phys Lett 72B (1978) 477,

L Susskind, Phys Rev D20 (1979) 2610

[4] M Creutz, Phys Rev D21 (1980) 2308; Phys Rev Lett 45 (1980) 313

[5] L McLerran and B Svetıtsky, Phys Lett 98B (1981) 195; Phys Rev D24 (1981) 450

[6] J Kutı, J Polonyı and K Szlachányı, Phys Lett 98B (1981) 199

[7] J Engels, F Karsch, I Montvay and H Satz, Phys Lett 101B (1981) 89

[8] K Kajant1e, C Montonen and E Pietarnen, Z Phys C9 (1981) 253

[9] I Montvay and E Pretarinen, Phys Lett 110B (1982) 148

[10] J Engels, F Karsch, I Montvay and H Satz, Phys Lett 102B (1981) 332

[11] K Wilson, Phys Rev D10 (1974) 2445

[12] E Mannan, G Pansı and C Rebbi, Phys Rev Lett 47 (1981) 1795 ,

H Hamber and G Parisi, Phys Rev Lett 47 (1981) 1792,

A Hasenfratz and P Hasenfratz, Phys Lett 104B (1981) 489;

D Weingarten, Monte Carlo evaluation of hadron masses in lattice gauge theories with fermons, Indiana University preprint IUHET-69 (Oct, 1981)

[13] C Bernard, Phys Rev D9 (1974) 3312

[14] P Pfeuty, Ann of Phys 57 (1970) 79

[15] C J Hamer and M N Barber, J Phys Al4 (1981) 241

[16] M Suzuk1, Progr Theor Phys 56 (1976) 1454

[17] E Fradkın and L Susskınd, Phys Rev D17 (1978) 2637

[18] F J Wegener, J Math Phys 12 (1971) 2259

[19] Y Y Goldschmidt and J Shigemitsu, Nucl Phys B200[FS4] (1982) 149

[20] S Elitzur, Phys Rev D12 (1975) 3978

[21] J Engels, F Karsch and H Satz, Nucl Phys B205[FS5] (1982) 239

[22] A Hasenfratz and P Hasenfratz, Nucl Phys B193 (1981) 210

[23] N S Manton, Phys Lett 96B (1980) 328,

P Menottı and E Onofr, Nucl Phys B190[FS3] (1981) 288,

C B Lang, C Rebb1, P Salomonson and B-S Skagerstam, Nucl Phys B190[FS3] (1981) 337

[24] F Karsch, Nucl Phys B205[FS5] (1982) 285

[25] J Kapusta, Nucl Phys B148 (1979) 461

[26] V F Muller and W Ruhl, Ann Phys 133 (1981) 240

[27] M Nauenberg, T Schalk and R Brower, Phys Rev D24 (1981) 548,

B Lautrup and M Nauenberg, Phys Rev Lett 45 (1980) 1755

[28] G Bhanot and C Rebbi, Nucl Phys B180[FS2] (1981) 469

[29] Dual Theory, ed M Jacob (North-Holland, Amsterdam, 1974)

[30] A Chodos, R L Jaffe, K Johnson and V Wesssopf, Phys Rev D9 (1974) 3471

[31] R Hagedorn, CERN report 71-12 (Yellow Senes), 1971

[32] K Huang and S Wernberg, Phys Rev Lett 25 (1970) 855,

H Satz, Phys Rev D20 (1979) 582

[33] N Cabibbo and G Parsi, Phys Lett 59B (1974) 67

[34] J Kogut, D.K Sinclair and L Susskınd, Nucl Phys B114 (1976) 199

[35] J Donoghue, K Johnson and B Li, Phys Lett 99B (1981) 416

[36] B Berg, Phys Lett 97B (1980) 401, G Munster, Nucl Phys B190[FS3] (1981) 439; (E, A B200[FS4] (1982) 536, E B205[FS5] (1982) 648)

[37] A D Linde, Phys Lett 96B (1980) 289;

A Billoire, G Lazandes and O Shafi, Phys Lett 103B (1981) 450 
[38] G Baym, Physica 96A (1979) 131,

T Celık, F Karsch and H Satz, Phys Lett 97B (1980) 128

[39] E V Shuryak, Phys Lett 107B (1981) 103,

R D Pisarskı, A mean field theory for the chiral phase transition in QCD, University of California preprint NSF-ITP-81-92 (1981) 